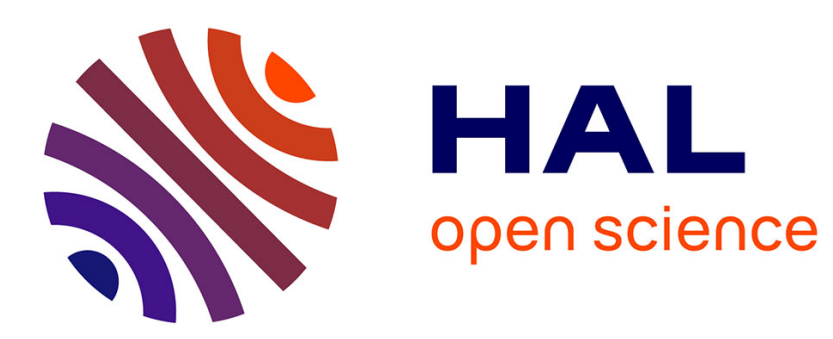

\title{
TOR inhibitors: from mammalian outcomes to pharmacogenetics in plants and algae
}

\author{
Marie-Hélène Montané, Benoît Menand
}

\section{To cite this version:}

Marie-Hélène Montané, Benoît Menand. TOR inhibitors: from mammalian outcomes to pharmacogenetics in plants and algae. Journal of Experimental Botany, 2019, 70 (8), pp.2297-2312. $10.1093 / \mathrm{jxb} / \mathrm{erz} 053$. cea-02073630

HAL Id: cea-02073630 https://hal-cea.archives-ouvertes.fr/cea-02073630

Submitted on 17 Feb 2020

HAL is a multi-disciplinary open access archive for the deposit and dissemination of scientific research documents, whether they are published or not. The documents may come from teaching and research institutions in France or abroad, or from public or private research centers.
L'archive ouverte pluridisciplinaire HAL, est destinée au dépôt et à la diffusion de documents scientifiques de niveau recherche, publiés ou non, émanant des établissements d'enseignement et de recherche français ou étrangers, des laboratoires publics ou privés. 


\section{TOR inhibitors: from mammalian outcomes to pharmacogenetics in plants and \\ 2 algae}

3 Review paper

4

$5 \quad$ Marie-Hélène Montané ${ }^{1 *}$ and Benoît Menand ${ }^{1 *}$

$6{ }^{1}$ Aix Marseille Univ, CEA, CNRS, BIAM, Laboratoire de génétique et biophysique des plantes,

7 Marseille, France F-13009

$8 *$ correspondance: marie-helene.montane@univ-amu.fr and benoit.menand@univ-amu.fr

9 Running title: TOR inhibitors in plant and algae

Keywords: TOR, mammals, plants, algae, rapamycin, ATP-competitive TOR inhibitor.
12

\section{Abstract}

Target Of Rapamycin (TOR) is a conserved eukaryotic phosphatidylinositol 3-kinase (PI3K)-related kinase (PIKK) that regulates growth and metabolism in response to environment in plants and algae. The study of the plant and algal TOR pathway largely depends on TOR inhibitors first developed for non-photosynthetic eukaryotes. In animals and yeast, fundamental works on the TOR pathway have benefited from the allosteric TOR inhibitor rapamycin and more recently from ATP-competitive TOR inhibitors (asTORis) that circumvent the limitations of rapamycin. The asTORis, developed for medical applications, inhibit TORC1 more efficiently than rapamycin and also inhibit rapamycin-resistant TOR complexes (TORCs). This review will present knowledge on TOR inhibitors from the mammalian field and underline important consideration for plant and algal biologists. We will discuss the use of rapamycin and 
asTORis in plants and algae and conclude with guidelines for physiological studies and genetic screens with TOR inhibitors.

\section{Introduction}

Rapamycin together with the structurally related drug FK506 are immunosuppressive agents that are reciprocal antagonists of lymphocyte cell activation (Sigal and Dumont, 1992). Rapamycin stood out for its role in second phase of lymphocyte activation by inhibiting cell cycle and subsequently proliferation (Aagaard-Tillery and Jelinek, 1994). A recent overview of rapamycin (Yoo et al., 2017) describes the respective mechanisms of immunosuppressive action of FK506 that interferes with the phosphatase calcineurin and of rapamycin that interferes with the serine/threonine kinase "Target Of Rapamycin" (TOR). Both compounds bind to a single domain of the cytosolic immunophilin FKBP12 (12 kDa FK506 Binding Protein). Briefly, the FKBP12-rapamycin duo binds to the so-called FRB (FKBP12-Rapamycin Binding) domain of TOR therefore creating a ternary complex that inhibits TOR kinase activity through allosteric interaction. Throughout eukaryotes, TOR progressively emerged as a hub for orchestrating cellular anabolic and catabolic processes that basically characterize growth homeostasis, i.e., cell/organ size and cell proliferation as well as cell components turnover. "In simple terms, cell growth is the accumulation of mass. But this description short changes a process that is vastly more complex and interesting" (Thoreen, 2017). TOR interconnects numerous inputs and outputs of anabolism functions while repressing autophagy, ensuring growth homeostasis, i.e, the building up, the "stability" or survival of cells up to their aging and senescence or in response to any imbalance caused by stress, disease or energy changes (Saxton and Sabatini, 2017; Thoreen, 2017). Rapamycin was decisive for the discovery of TOR protein, basic TOR complexes (TORCs) components and targets (Alessi et al., 2009; Huang et al., 2003), yet the recent development of ATPcompetitive TOR inhibitors (active site TOR inhibitors, as TORis) brought new tools to study more in 
depth the TOR pathway. Furthermore, since the treatment of cancer by rapamycin and its derivatives rapalogs gave disappointing results, these second generation inhibitors also provided new possibilities of clinical trials aiming to cure cancer and other pathologies (Martelli et al., 2018). In the context of studying TOR functions in plants, we aim to state here the use of rapamycin and of asTORis with an emphasis on their potential for pharmacogenetic studies in plant and algae.

\section{Rapamycin and TOR complexes from yeast and mammals to plants and algae}

For the historical steps on the discovery and naming of TOR, we invite the reader to rely on very informative articles of DA Sabatini and MN Hall (Hall, 2016; Sabatini, 2017). The TOR protein kinase was first identified from a genetic screen of Saccharomyces cerevisiae (referred hereafter as yeast) lines that were resistant to rapamycin (Heitman et al., 1991). Rapamycin-resistant lines mostly carried recessive missense mutations resulting in amino acid substitutions in the FKBP12 protein, but dominant missense mutations in two genes named TOR1 and TOR2 (Target Of Rapamycin 1 and 2) were also identified. Further studies revealed that mutations of a conserved Serine residue within the FRB domain of TOR1 or TOR2 confers dominant resistance to rapamycin (Stan et al., 1994). Soon after, three groups identified the "physical target of rapamycin" in mammals by biochemical approaches using rapamycin and FKBP12. TOR is a member of the atypical Ser/Thr-protein kinase of the PIKKs family that all play vital role in growth and survival and also includes essential regulators of the DNA damage response such as ATM (Ataxia-Telangiectasia Mutated), ATR (ATM- and Rad3Related) and DNA-PK (DNA-dependent Protein Kinase) (De Cicco et al., 2015). Rapamycin has been an indispensable tool for studying the roles of the TOR pathway in both yeast and animals but rapamycin effects are more limited in animals on protein synthesis, autophagy and proliferation (Fig. 1A) and varied widely among cell types (Mukhopadhyay et al., 2016; Sarbassov et al., 2006; Thoreen, 2017; Zhao et al., 2015). Both genetic and biochemical studies identified two basic TOR complexes: The rapamycin sensitive TORC1 containing RAPTOR/KOG1 (mammalian Regulatory Associated 
71 Protein of TOR/yeast Kontroller Of Growth 1) and LST8 (Lethal with SEC13 protein 8), and the

rapamycin-insensitive TORC2 containing LST8 and RICTOR/AVO3 (mammalian RapamycinInsensitive Companion of mTOR/yeast Adheres-VOraciously-to-tor-2 protein 3). TORC2 components and downstream effectors have been difficult to characterize due to the absence of specific drugs that selectively inhibit this complex (Gaubitz et al., 2016; Sparks and Guertin, 2010) and because under prolonged (chronic and not acute) rapamycin treatment, TORC2 assembly was impaired (Sarbassov et al., 2006). TORC2 is involved in cell survival and cytoskeleton regulation through different AGC family kinases including a key readout target kinase AKT, which phosphorylation requires SIN1/AVO1 (mammalian Stress-activated protein kinase-INteracting protein 1/yeast AdheresVOraciously-to-target-of-rapamycin-2 protein 1), another essential component of TORC2 (Gaubitz et al., 2016). Noticeably, SIN1 isoforms led to suggest occurrence of 3 different TORC2, showing plasticity of TOR complexes. A detailed composition of yeast and mammals TORC1 and TORC2 and the full range of downstream targets through which TOR drives cell growth has recently fully emerged and is extensively reviewed elsewhere (Ben-Sahra and Manning, 2017; Eltschinger and Loewith, 2016; Gaubitz et al., 2016; Gonzalez and Rallis, 2017; Jhanwar-Uniyal et al., 2017; Saxton and Sabatini, 2017).

The control of cell growth by TORC1 in response to nutrients was early demonstrated in yeast (Barbet et al., 1996) and later on transcriptional profiling showed that mammalian TORC1 upregulates sets of genes involved in lipid/sterol, nucleotide and protein synthesis, as well as genes involved in mitochondrial oxidative function, glycolysis and the pentose phosphate pathway and conversely down-regulated genes involved in starvation and energy production (Duvel et al., 2010; Peng et al., 2002). Briefly, under adequate conditions including growth factors, amino acids and AMP to ATP and/or ADP to ATP ratios, TORC1 phosphorylates two foremost targets involved in protein synthesis commitment and elongation, the eIF4E-Binding Protein1 (4E-BP1) and the ribosomal protein S6 Kinases (S6Ks) respectively. However, it is worth mentioning that the clear cut contribution 
of each TORC1-S6K1/S6K2 and TORC1-4E-BP1 axis in regulating cell cycle and proliferation as well as translation has been hard to delineate (Cunningham et al., 2007; Dowling et al., 2010a; Magnuson et al., 2012; Meyuhas and Dreazen, 2009; Thoreen, 2017). First, their kinetics of phosphorylation do not last the same and their different action in the regulation of protein synthesis machinery, which involves additional TOR targets, made it complex to decipher (Dowling et al., 2010a; Magnuson et al., 2012; Meyuhas, 2015; Thoreen, 2017). As such, the TORC1 target LARP1 (La-Related Protein 1) is a translation repressor that, according to a recent model, binds to the 5' end of mRNAs and thus competes with the translation initiation complex eIF4F (including eIF4E) and to some extent with S6K1 (Philippe et al., 2018). TORC1 controls protein turnover through regulating UPS (Ubiquitin Proteasome System)- and UPS targeted-proteins abundance (Rousseau and Bertolotti, 2016; Zhao et al., 2016) as well as by canonical autophagy induction through regulating activity of the kinases ULK1 and 2/ATG1 (Human Uncoordinated-51-like autophagy activating kinase 1and 2/yeast AuTophaGy related 1) (Velazquez and Jackson, 2018; Zhao et al., 2015). Remarkably, due to reversible control of ULK1 by mTOR and AMP-activated Protein Kinase (AMPK) that senses low energy levels, mammalian growth homeostasis is orchestrated through dynamic signaling interplay of the triad of kinases, AMPK-TOR-ULK1. Under low energy, if ULK1 is activated through phosphorylation by AMPK, it can be impeded by TORC1 and in turn, ULK1-mediated phosphorylation can decrease activity of AMPK, establishing a negative feedback loop targeting the AMPK-mTOR signaling axis (Dunlop and Tee, 2013; Luo et al., 2015). Also, a positive regulation loop occurs through phosphorylation by TOR and ULK1 of a component in autophagosome formation, which joins regulation of effectors by phosphorylation to regulation by ubiquitylation (Nazio et al., 2013).

More extensively, the crosstalk between different branches of the TOR network is nowadays upgraded by the emerging view that negative feedback loops where downstream targets become upstream regulators might be critical in the TOR pathway (Eltschinger and Loewith, 2016). As such, 
the negative feedback loop of the TOR-S6K-IRS1 (Insulin Receptor Substrate 1) axis in response to TORC1 activation that is mediated by S6K1 attenuates PI3K-AKT signaling by phosphorylating IRS1 and RICTOR leading to AKT kinase inhibition. These two examples of feedback loops state the importance of characterizing cell developmental or metabolic status when deciphering the role of specific TOR pathway effectors as physiology "customizes" TOR signaling backbone status. For instance, in the field of TOR-driven aging, cell entry into senescence is decelerated by rapamycin, preventing irreversible loss of proliferation capacity through inhibiting the senescence-associated secretory phenotype of cells without affecting cell cycle arrest (Wang et al., 2017). This led defining new concepts and so new terms in order to delineate clear-cut functions of effectors in cell cycle arrest and/or senescence (Blagosklonny, 2012). Another important feature is that TOR basic targets S6Ks, 4E-BPs, ULK1 or components of TOR complexes (SIN1, RAPTOR, RICTOR) very often carry multiple phosphorylation sites, which likewise helps connecting different signaling pathways to the TOR pathway to maintain cell homeostasis but makes analysis more complex (Batool et al., 2017; Meyuhas, 2015; Tavares et al., 2015). At last but not least, the recent discovery of new TOR complexes that do not contain RAPTOR or RICTOR reveals the extent of TOR function. As such, a complex TOR-RanBP2 (Ran Binding Protein 2) that ensures dynamic flux of nuclear import of ribosomal proteins (Kazyken et al., 2014), a complex TOR-GIT1 (G-protein-coupled receptor kinaseinteracting protein 1) essential for astrocyte survival (Smithson and Gutmann, 2016), a rapamycin insensitive TORC3 including at least LST8 and an unknown protein phosphorylating mSIN1 (Luo et al., 2015), or a new rapamycin sensitive TORC acting on mRNA translation (Meyuhas, 2015) have been identified. Another cytoplasmic TORC3 activated in cancer solely contains mTOR, 4E-BP1 and the transcription factor ETV7 (leukemia virus E26 Transformation-specific Variant 7) but not the TORC1/2 crucial components LST8, RAPTOR, RICTOR or SIN1 (Harwood et al., 2018). TOR complexes can have various intracellular localization, close to either the nucleus or the perinuclear region, lysosomes, mitochondria-associated endoplasmic reticulum membranes or plasma membrane 
depending on nutrient status (Betz and Hall, 2013; Jhanwar-Uniyal et al., 2017). This also holds true for the target S6K (Tavares et al., 2015) and altogether this makes TORCs eclectic, in coherence with the role of TOR in cell growth homeostasis. Altogether, the discovery of new TORCs, their diverse intracellular localization, the interaction of TOR- and other- signaling pathways and the multiple phosphorylation sites of TOR pathway components reflect the deployment of TOR signaling and the importance to decipher its role in clearly defined cellular contexts.

In plants, early studies benefited from the conservation of TOR among species and the libraries of Arabidopsis insertion mutants which helped find knock-out mutants of homologs of yeast and mammalian genes encoding basic members of TORCs. Thus, Arabidopsis genome contains one TOR gene (AtTOR) (Menand-2002), two RAPTOR genes (Anderson et al., 2005; Deprost et al., 2005; Mahfouz et al., 2006; Rexin et al., 2015; Salem et al., 2018) and two LST8 genes (Moreau et al., 2012). Arabidopsis raptor mutants are still under study and sporadic embryonic arrest has been controversial likely due to poor quality of some insertion mutants (Rexin et al., 2015), making it different from mammals where RAPTOR ablation is associated with male sterility (Xiong et al., 2017b). LST8s function is still in progress since only lst8-1 mutant phenotype and not lst8-2 is documented, yet altered growth and particularly metabolomic phenotype of lts $8-1$ reminds amino acid accumulation observed in yeast $l s t 8$ mutants (Moreau et al., 2012). Thus, in the absence of RICTOR homologs, only basic TORC1 is characterized in plants and algae until now (Dobrenel et al., 2016a; Perez-Perez et al., 2017; van Dam et al., 2011). The main plant TOR targets include S6K1 and S6K2, which are both related to mammalian S6K1, and the PP2A (Protein Phosphatase 2A) regulatory subunit TAP46 (Ahn et al., 2011; Henriques et al., 2010; Mahfouz et al., 2006; Xiong and Sheen, 2012). TOR negatively regulates autophagy also in plants and green algae (Liu and Bassham, 2010; Perez-Perez et al., 2010) and even though convergence of UPS and autophagy has been demonstrated in plants (Marshall et al., 2015) TOR dependent regulation of UPS is still unknown. Strikingly, the catalytic subunit KIN10 of SnRK1 (Snf1-Related protein Kinase 1), the plant homolog of mammalian 
AMPK/yeast SNF1, regulates autophagy through inhibiting TOR and SnRK1 is not regulated by the AMP/ATP ratio similarly to yeast SNF1 and contrarily to mammalian AMPK (Soto-Burgos and Bassham, 2017). The position of the ATG1/13 kinase complex in autophagy is also central in plants with four isoforms of the ATG1 kinase, two of its partner ATG13 (Suttangkakul et al., 2011) and accessory ATG proteins such as ATG11 (Li and Vierstra, 2014) reported in Arabidopsis, yet ATG1 phosphorylation by TOR has not been demonstrated (Wang et al., 2018a). Thus to control autophagy in plants, TOR might target ATG13 to regulate ATG1 similarly to yeast (Kamada et al., 2010) rather than regulating both ATG13 and ULK1 as in mammals (Kim et al., 2011). Interestingly, Arabidopsis ATG1 has a dual role through acting as a regulator and as a substrate of autophagy, likely a particular feature of plants (Bassham, 2009; Suttangkakul et al., 2011). However, as plant autophagy effectors and processes are still under study (Masclaux-Daubresse et al., 2017; Wang et al., 2018a), this field requires more investigation. Other plant TOR targets were also identified (Shi et al., 2018), including the transcription factors $\mathrm{E} 2 \mathrm{FA}$ and $\mathrm{E} 2 \mathrm{FB}$ which phosphorylation in vitro is lost by treatment with ATP-competitive inhibitors (Torins, see below) (Li et al., 2017; Xiong et al., 2013), or the hormone abscisic acid-receptor PYL1 (PYrabactin resistance 1-Like 1), which activity is associated with stress and senescence (Wang et al., 2018b). In the absence of plant homologs of 4E-BP1, the axis TORC1S6Ks is nowadays the most studied link between TOR and translation in plants, mainly through read out of ribosomal protein S6 phosphorylation (Dobrenel et al., 2016b; Mahfouz et al., 2006; Xiong and Sheen, 2012). However, the recent discovery of a Conserved Binding of eif4E1 (CBE1) plant protein (Patrick et al., 2018) opens new possibilities of link between TOR and translation initiation in plants. In Chlamydomonas reinhardtii, recent phosphoproteomic studies identified TOR-inhibition dependent phosphorylation of proteins including ATG7, S6K, the ribosomal protein S6 and LARP1 (Roustan and Weckwerth, 2018; Werth et al., 2018) showing conservation of effectors in algae and opening new avenues of TOR pathway characterization. In the red alga Cyanidioschyzon merolae, a phosphoproteomic analysis with the a transgenic strain overexpressing yeast FKBP12 identified 
GLG1, an authentic GLycoGenin which phosphorylation is cancelled by rapamycin (Pancha et al., 2018). As systems biology and omics now start connecting TOR pathway with specific aspects of plant and algae physiology, new TOR targets could be discovered soon in photosynthetic organisms (Caldana et al., 2013; Dobrenel et al., 2016a; Mubeen et al., 2018). New TOR complexes might exist in plants and algae, as discovered in animals, but their future identification would need more specific biochemical or genetic studies. Altogether, these data show that the TOR pathway includes conserved and specific effectors in photosynthetic organisms and thus its study benefits from outcomes from yeast and mammalian studies, as well as from plant and/or algae specific investigations.

\section{Rapamycin-FKBP12 -TOR inhibition in plants and algae: not an easy game}

In algal species, rapamycin sensitivity is species-dependent and growth inhibition level (GI \%) and doses $(\mathrm{nM})$ are highly variable as they range from $(40 \% ; 100 \mathrm{nM})$ for Chlamydomonas reinhardtii (Crespo et al., 2005), to (40\%; 50,000 nM) for Euglena gracilis (Mukaida et al., 2016), to (slight effect; $10,000 \mathrm{nM}$ ) for the diatom Phaeodactylum tricornutum (Prioretti et al., 2017) up to (0\%; 1,000 nM) for the red algae C. merolae (Imamura et al., 2013). However, chlorophyll content decreased in $E$. gracilis and $C$. merolae from 1,000 nM but not in C. reinhardtii (Mukaida et al., 2016). A rapamycin resistant FKBP12 loss-of-function mutant in C. reinhardtii allowed to demonstrate that rapamycin inhibits proliferation via the rapamycin-FKBP12 interaction, a strong argument for the further use of rapamycin in this alga (Crespo et al., 2005). These few data show that rapamycin is not a general potent TOR inhibitor in algae species, reminding the variety of background responses of mammalian cell lines. In vascular plants, rapamycin hardly inhibits growth of various genera including Arabidopsis, Nicotiana, cotton or potato plantlets with some peculiar cases like tomato where partial growth inhibition has been observed (Deng et al., 2017; Deng et al., 2016; Mahfouz et al., 2006; Menand et al., 2002; Montane and Menand, 2013; Ren et al., 2012; Song et al., 2017; Sormani et al., 2007; Xiong et al., 2016). Insensitivity or weak sensitivity to rapamycin has been attributed to low 
ability of plant FKBP12 proteins to form the inhibitory ternary complex with rapamycin due to lack of conservation of aminoacid residues critical for interaction with rapamycin (Supplementary Fig. S1) (Choi et al., 1996; Sormani et al., 2007; Xu et al., 1998). A similar situation was described in red algae (Imamura et al., 2013). There is no straightforward evolutionary explanation for this particular feature of FKBP12s of plants and some algae, but we could speculate the selection of new FKBP12 endogenous peptidyl-prolyl isomerase functions or a selective advantage to resist to the soil Streptomycete that produces rapamycin (Vezina et al., 1975). Neither the yeast two-hybrid analysis nor an in vitro interaction assay could demonstrate a rapamycin-dependent interaction between Arabidopsis FKBP12 (AtFKBP12) and the AtTOR-FRB domain but this domain was able to form a complex with human (Hs) or yeast FKBP12 (Mahfouz et al., 2006; Menand et al., 2002; Sormani et al., 2007). As a consequence, Arabidopsis plants could be made sensitive to rapamycin by overexpression of yeast or human FKBP12 (Deng et al., 2016; Leiber et al., 2010; Ren et al., 2012; Sormani et al., 2007; Xiong and Sheen, 2012). A yeast-FKBP12 overexpressing line in the red alga $C$. merolae similarly confers sensitivity to 10-500 nM rapamycin (Imamura et al., 2013). However, to our opinion, the dogma of plant TOR kinase inhibition by rapamycin through transgenic FKBP12 overexpression deserves little bit more attention.

Several groups reported that Arabidopsis seedlings grown on solid media are insensitive to rapamycin up to ca. $10 \mu \mathrm{M}$ (Deng et al., 2016; Mahfouz et al., 2006; Ren et al., 2012; Sormani et al., 2007). Such concentration range is $100-1000$ times the concentration that inhibits proliferation of yeast (100 nM block cells in G1 with large unbudded cells as the terminal phenotype (Heitman et al., 1991) or that reduces cell size and proliferation of lymphocytes B cells (EC50 0.005-0.5 nM and maximal inhibition of ca. 50-70\% up to $100 \mathrm{nM}$ ) or of mouse embryonic fibroblasts (50-250 nM) (Sarbassov et al., 2006; Thoreen and Sabatini, 2009; Wicker et al., 1990). Later, AtTOR-dependent phosphorylation at P-T449 (equivalent to $\mathrm{T} 389$ in animal) of AtS6K1 overproduced in transfected protoplasts was found inhibited by far much lower concentrations of rapamycin when FKBP12 was co-expressed 
compared to the AtS6K alone. Indeed, 100 to 1000 times lower concentration of rapamycin was needed to erase S6K1 P-T449 when S6K1 was overexpressed in combination with either AtFKBP12 or HsFKBP12 respectively (Xiong and Sheen, 2012). This showed that a high amount of HsFKBP12 "optimize" the titration of rapamycin to inhibit plant TOR. Thus, the affinity to rapamycin and the stoichiometry of each component of the ternary complex might influence the stability of TOR complex conformation shift and therefore the outcome of TOR inhibition. In other words, the poorest the interaction FKBP12-rapamycin is, the highest the rapamycin concentration is required to erase AtS6K1 P-T449. Thus, if we consider that this is the rule despite the peculiar physiological context of protoplasts incubated in mannitol and $\mathrm{KCl}$ in which it has been studied (no nutrients), a low amount of AtFKBP12 together with a poor binding of endogenous AtFKBP12 to AtTOR can explain why plants are poorly sensitive to rapamycin. Deng et al. similarly developed transgenic plants overexpressing FKBP12 coming from Arabidopsis, yeast and human but showed that AtFKBP12 overexpression could not make plants sensitive to rapamycin (Fig. 1C) (Deng et al., 2016). This discrepancy with the data of Xiong and Sheen (Xiong and Sheen, 2012), shows the possible drawback of building transgenic lines to overexpress FKBP12 (FKBP12 $\left.{ }^{\mathrm{OX}}\right)$. Yet, the overexpressed yeast FKBP12 was more efficient than HsFKBP12 to increase plant sensitivity to rapamycin (Fig. 1C) (Deng et al., 2016).

Growth conditions also influence rapamycin sensitivity as Arabidopsis seedlings grown in liquid culture were reported sensitive to rapamycin (Deng et al., 2016; Xiong et al., 2013; Xiong and Sheen, 2012). An interesting explanation for the discrepancy between plants grown on liquid and solid media was proposed by M Ren and colleagues who suggested that a hypoxia stress could facilitate rapamycin action in Arabidopsis (Deng et al., 2016). Indeed, growth is dramatically slowed down in liquid media as after 9 days, seedlings roots were around 2-3 cm long (Xiong and Sheen, 2012), which is around 2-3 times less than vertically grown seedlings on solidified medium (Montane and Menand, 2013; Ren et al., 2012). It is therefore likely that hypoxic stress might upregulate AtFKBP12 expression as FKBPs are reported to have a role in stress response (Dong et al., 2018; Geisler and 
Bailly, 2007) and/or that AtTOR is largely inhibited in this condition which might make AtTOR activity easier to be inhibited by rapamycin. Interestingly, the sensitivity of Arabidopsis seedlings to the rapamycin structurally related compound FK506 (that binds FKBP12 but not TOR), was tested in WT and yeast-FKBP12 ${ }^{\text {OX }}$ lines (Zhang et al., 2013). The authors concluded that FK506 has no effect on seedlings growth but the seedling phenotype shown after 25 days on half strength Murashige and Skoog (MS) medium containing $20 \mu \mathrm{M}$ FK506 appears to us different from the control. Indeed, roots hardly grew in the solid medium but grew in the air outside the solid matrix, a feature already observed after 9 days on non-optimal full strength 1xMS medium (Ren et al., 2012). This altered growth is characteristic of a stress due to non-optimal or to toxic drug containing medium and might reveal potential TOR-rapamycin-independent phenotypes. Thus, we think that more detailed analysis is required before ruling out that FK506 affects or not the physiology of yeast-FKBP12 ${ }^{\text {OX }}$ lines.

Additionally, Arabidopsis FKBP12 was shown to interact with a nuclear protein that controls endoreduplication and therefore might control this process as in mammals (Vespa et al., 2004). As in the case of calcineurin, HsFKBP12 is also a subunit of the transforming growth factor B (TGF$\beta$ ) type I receptor, a transmembrane Ser/Thr kinase that regulates cell growth and differentiation (Gold et al., 1997). HsFKBP12-- cells show cell cycle arrest due to impaired regulation of TGF- $\beta$ receptor signaling (Aghdasi et al., 2001). Thus, it appears relevant to wonder whether in the absence of rapamycin AtFKBP12 is involved in cell cycle regulation or other signaling regulations that might interfere with TOR signaling studies with FKBP12 ${ }^{\mathrm{OX}}$ lines. Indeed, expressing an heterologous FKBP12 gene might also change plant physiology as the PaFKBP12 gene from the Antarctic moss Polytrichastrum alpinum ectopically expressed in Arabidopsis increases plant stress tolerance (Alavilli et al., 2018). Thus, even though FKBP12 ${ }^{\mathrm{OX}}$ lines do not have a macroscopic phenotype (Deng et al., 2016; Sormani et al., 2007), it does not preclude conditional cell/tissue responses compared to WT. Not least, when regarding the other component of the duo FKBP-Rapamycin, a concentration range of 0.5-5 $\mu \mathrm{M}$ rapamycin was shown to interfere with interactions of the core particle 20S with its cellular 
activators and consequently to inhibit proteasome by attenuating major peptidase activities (Osmulski and Gaczynska, 2013). The authors hypothesized that interactions of the proteasome with rapamycin induce a maximal conformation shift of the proteasome, which results in compromised gating of substrates. Such an off target of rapamycin might interfere with other functions independently of the effects of TOR inhibition. Whether proteasome is an off target of rapamycin also in plants is unknown but should be carefully considered as proteolysis is enhanced following TOR inhibition (Zhao et al., 2015) and UPS and autophagy converge in Arabidopsis (Marshall et al., 2015). At last, another hint is the putative role of metabolites interacting with TOR such as phosphatidic acid that might impede interaction rapamycin-TOR and explain the strong variation of rapamycin dose to inhibit TOR in different mammalian cell lines (Mukhopadhyay et al., 2016). Anyhow, altogether this underscores that FKBP12 and/or rapamycin dosage as well as the cell physiology might be carefully controlled as they influence TOR inhibition-dependent results. This also opens the question of the selectivity of rapamycin. Therefore, compared to yeast and mammals, the conditional FKBP12 overexpressiondependent allosteric inhibition of TOR by rapamycin in plants might easily turn to be a conundrum.

Another aspect to underline is that for each combination of FKBP12 $2^{\mathrm{OX}}$ lines, the dose response to rapamycin shows that growth cannot be completely inhibited. Seedlings growth of yeast-FKBP12 ${ }^{\text {OX }}$ lines was partially inhibited by 1-20 $\mu \mathrm{M}$ rapamycin (Deng et al., 2016; Ren et al., 2012) or by even lower range of 10-100 nM (Zhang et al., 2013). Anyhow, routine rapamycin concentration ranges are 4-10 $\mu \mathrm{M}$ to inhibit such lines (Leiber et al., 2010; Sormani et al., 2007; Xiong and Sheen, 2012), which makes it hard to appreciate rapamycin potency in plants. However, growth inhibition of yeastFKBP12 ${ }^{\text {OX }}$ plants by rapamycin never exceeds a plateau value of ca. 50\% (Fig. 1C), which reminds the incomplete efficacy of rapamycin action observed in mammals (Fig. 1A and C). Similarly, in the green algae $C$. reinhardtii, which is naturally sensitive to rapamycin (Supplementary Fig.S1) in both solid and liquid media, maximal growth inhibition was also ca. 50\% (Fig. 1E) (Crespo et al., 2005; Juppner et al., 2018; Roustan and Weckwerth, 2018). Therefore, in both yeast-FKBP12 ${ }^{\mathrm{OX}}$ plant lines 
and rapamycin-sensitive WT algae challenged till now, full growth inhibition cannot be reached with rapamycin (Fig. 1C and D), making rapamycin efficacy not maximal. With that in mind, we can conclude that rapamycin can be used with more confidence in Chlamydomonas reinhardtii than in plants and other algae as, even if TOR inhibition is probably partial, transgenic over-expression of FKBP12s is avoided.

\section{Inhibition of TOR by ATP competitive inhibitors: a new deal}

Within this highly dynamic research field, studies of TOR kinase have considerably increased over the last 10 years and limitations of allosteric rapamycin- and rapalogs-based clinical strategies have pushed toward the development of orthosteric ATP-competitive mTOR inhibitors that were called asTORis (active site TOR inhibitors), TORKis or TKIs (TOR Kinase Inhibitor) (Martelli et al., 2018). In contrast to rapamycin, they target the kinase domain of mTOR and are able to fully inhibit mammalian TORC1 activity in a dose dependent manner but also TORC2 and other TOR complexes (Fig. 1A and B) (Chresta et al., 2012; Dowling et al., 2010b; Harwood et al., 2018; Kang et al., 2013).

Here we would like to remind that the terms of potency, efficacy, selectivity, metabolic stability, off rate (also called associated residence time), and off targets as well as pharmacokinetic characteristics of a drug at the organism level (PK), altogether define a drug singularity. Potency and efficacy are parameters that are derived from graded dose-effect curves and that can be used to compare drugs that elicit the same pharmacological effect (Mosby's Medical Dictionary, 2009).

Potency, which is a measure of the sensitivity of a target organ or tissue to a drug, is a relative term that relates the amount of one drug required to produce a desired level of effect to the amount of a different drug required to produce the same effect. On the semi-logarithmic graded dose-effect plot, the curve of the most potent agent tends to be in the left side of the graph and the median effective 
concentration (EC50) is lower. A drug's potency is influenced by its affinity for its receptor and therefore independent of its maximal effect. Efficacy (or intrinsic activity) is the drug property that allows the receptor-bound drug to produce its pharmacological effect. The relative efficacy of two drugs that elicit the same effect can be measured by comparing the maximum effects of the drugs. A drug can have high potency but poor efficacy, meaning that the response is seen at very low doses and remains small even at high doses. This is the case of rapamycin which is a highly potent but poorly efficient TOR allosteric inhibitor (Fig. 1A, C and E) compared to active site TOR inhibitors which are highly efficient (Fig. 1B, D and F). If a drug has one effect, and only one effect on all biological systems it possesses the property of specificity. In experience, the vast majority of drugs are selective rather than specific (Davis et al., 2011). "A drug with the appropriate balance of avoidance of undesirable targets (narrow selectivity) and coverage of one or more targets of interest (broad selectivity) is a continual drug development challenge. In many cases this objective is attained through trial and error, but there are rational approaches that can guide the tuning of selectivity, and examples have been published that illustrate a number of generalizable strategy" (Huggins et al., 2012). Thus, "a Selectivity score (S) for each drug can be calculated by dividing the number of kinases found to bind with dissociation constant $<3 \mu \mathrm{M}$ " (or sometimes $10 \mu \mathrm{M}$ ) "by the total number of distinct kinases tested. The selectivity score is an unbiased measure that enables quantitative comparisons between compounds and the detailed differentiation and analysis of interaction patterns" (Karaman et al., 2008).

At last, in pharmacology, an inhibiting or effective concentration (IC or EC) refers to a concentration of a drug that produces a biological response in case of enzymology in vitro assays or when unicellular organisms or mammalian cell cultures are tested. IC refers to an assay where there is decrease in activity whereas EC rather refers to a drug that activates a system. The term effective dose (ED) refers to in vivo studies when used in living organisms such as animals to usually determine the median effective dose (ED50) and/or the median lethal dose (LD50). Usually, in the context of studies 
involving asTORis, potency values obtained by means of in vitro enzyme-based assays $\left(\mathrm{IC}_{\text {in vitro }}\right)$ are generally different than potency values $\left(\mathrm{IC}_{\text {cell }}\right)$ obtained by cell-based assays treating cells before measuring various enzymatic products (also called cell potency values) because many targets can be modified at more than one phosphorylation site or in more than one way, e.g., ubiquitylation or acetylation (Carlson et al., 2009). Dissecting biochemical effects using in vitro grown cell lines might also give rise to different EC values depending on the cell physiology/line and is a far much different task than looking for clinical outcomes. Indeed the efficient doses (ED) are usually higher than those of in vitro studies likely to encompass drawbacks linked to PK properties, metabolic stability and putative off targets effect in organisms. A tool such as KInhibition portal (https://kinhibition.fredhutch.org) might help choosing a set of selective drugs among thousands depending on the objective (Bello and Gujral, 2018). Thus, designing a scale of inhibitor "strength" of a set of inhibitors solely from enzymatic properties (in vitro IC50) to calibrate experiments with living cells or organisms might be hazardous (Michel and Seifert, 2015).

Chemical structure activity relationship through docking studies using the TOR kinase domain with the dual PI3K/PIKK inhibitor NVP-BEZ235 (BEZ235), the TOR selective inhibitor PP242, and the TOR specific inhibitor KU-0063794 showed that drugs in development utilize a novel pharmacophore space to achieve specificity of TOR inhibition (Sturgill and Hall, 2009). So around the year 2009, several compounds were reported as asTORis (Fig. 2): PP242 (Feldman et al., 2009), Torin1 (Liu et al., 2010; Thoreen et al., 2009), KU-0063794 (Garcia-Martinez et al., 2009), WYE-354, 600 and 687 (Yu et al., 2009) and others reviewed by Benjamin and colleagues (Benjamin et al., 2011). These compounds were generally developed from dual PI3K/PIKK inhibitors or inhibitors more largely involved in the PI3K/AKT axis and have different core structure (Andrs et al., 2015; GarciaEcheverria, 2011; Liu et al., 2012a). They were TOR selective, having IC50 for TOR lower than for PI3Ks and also for other PIKKs (Benjamin et al., 2011). For instance, Torin1 efficiency towards TOR was compared to the effect of the dual PI3K/PIKK inhibitors PI103 and BEZ235 and its high 
selectivity towards a panel of kinases including PI3Ks and PIKKs was shown. Torin1 has a quinolone core structure expected to share the same binding mode as BEZ235 with mTOR, PI3K and other PIKK family members while PP242 has a pyrazolopyrimidine core structure derived from PP2 (Feldman et al., 2009). KU-0063794 and WYE-354 also derive from other dual PI3K/PKK inhibitors such as PI103 and LY294002 and contain a morpholino-substituted heterocycle. These asTORis were reported more efficient than rapamycin by measuring their capacity to phosphorylate TOR targets and also inhibit cell proliferation more efficiently. So new TORC1-dependent functions and previously found rapamycin resistant were deciphered due to higher efficacy of asTORis (Feldman et al., 2009; Thoreen and Sabatini, 2009) leading to new advances in TOR pathway knowledge (Guertin and Sabatini, 2009). For instance, the more effective TOR inhibition unveiled rapamycin resistant levels of regulation in cap-dependent initiation of translation, protein synthesis and proliferation (Dowling et al., 2010b). This also helped show that mTOR activates cap-dependent translation of cyclins and represses cap-independent translation of p27/KIP1, an inhibitor of CDK (Cyclin Dependent Kinase), therefore activating cell proliferation (Thoreen et al., 2009). These differential effects of rapamycin on substrates phosphorylation compared to that of the ATP competitive inhibitor Torin1 were studied through designing peptides from well-known TORC1 targets containing phosphorylation sites (Kang et al., 2013). When Torin1 blocks the phosphorylation of all TORC1 dependent phosphorylated sites in all TOR protein targets, some are not dephosphorylated by rapamycin (called strong target) and some are (called poor target). This concept of substrate quality is a property of TOR effector sites, which can explain that their differential phosphorylation vary with growth conditions. Furthermore, poor and strong targets can be found in the same protein (Fig. 3). In parallel, strong targets were also found phosphorylated in cells growing under partially depleted nutrient conditions (Fig. 3). Hence, rapamycin can be highly potent and selective for some poor mTOR targets such as S6K T389 and 4EBP1 S65, but its intrinsic activity or efficacy cannot be maximal since TOR is still able to phosphorylate the strong mTORC1 targets such as 4E-BP1 T37/46 and ULK1 S758 in presence of 
rapamycin (Kang et al., 2013). Therefore, due to the incomplete intrinsic efficacy, rapamycindependent TOR inhibition by an acute dose might lead to error-prone interpretation of data especially when targets are not well identified and/or when a chronic dose of rapamycin is applied (Sarbassov et al., 2006).

A comparison of selectivity, potency and metabolic stability of four asTORis mentioned above carrying different structure, i.e., Torin1, PP242, WYE-354 and KU-0063794 (Fig. 2) was reported and it appears to us to be a good example of how selectivity of a drug to TOR is demonstrated through tests involving many different assays (Liu et al., 2012a). They all exhibited highly potent and similar IC50 values against the recombinant mTOR kinase domain but their relative cellular potency EC50 against the TORC1 complex was: Torin1 > KU-63794 > WYE-354 > PP242. Their relative selectivity score toward a panel of 442 kinases was: KU-0063794 > WYE-354 > Torin1 > PP242. Other kinase assays showed that Torin 1 concentration above $1 \mu \mathrm{M}$ was able to inhibit the other PIKKs: ATM, ATR and DNA-PK. The metabolic stability was also better with KU-0063794 and WYE-354 than with Torin1 and PP242. However, Torin1 had a slower off-rate as the duration of S6K1 (pS6K-Thr-389) and PI3K-dependent (p-AKT-Thr-308) phosphorylation last 16 hours vs 1 hour for the 3 other drugs after extensive washing out the drug. Altogether, authors' conclusion of the study of these four asTORis was to avoid PP242 and to cautiously interpret data when Torin1, KU-0063794 and WYE354 are used at concentrations above $1 \mu \mathrm{M}$. Proliferation assays on mouse embryonic fibroblasts (MEFs) showed that in the range of $10-500 \mathrm{nM}$, rapamycin induces a plateau value of ca. $50-60 \%$ inhibition without any dose dependence, whereas Torin1 induces $40 \%$ to $100 \%$ inhibition between 10 and 250 nM when IC50 kinase values were 1-10 nM (Thoreen et al., 2009). Hence, Table 1 shows that IC50 values for in vitro TOR kinase activity do not fully predict the IC50 of proliferation. These differences likely deal with intrinsic PK, metabolic stability of the drug, or posttranslational modifications of the target as well as drug efflux or inactivation by cells as in yeast (Liu et al., 2012b).

Improvement of the pharmacokinetic properties of KU-0063794 led to the development of 
AZD-8055, which is very highly selective for mTOR over PI3Ks and PIKKs (Chresta et al., 2012;

Garcia-Echeverria, 2011; Marshall et al., 2011) and more recently to the less selective but more stable sister compound AZD2014 (Pike et al., 2013) (Fig. 2), both used in clinical trials (Garcia-Echeverria, 2011). A continuous development of intermediate compounds of this series that showed very high specificity towards PI3Ks although lower potency towards TOR led to the design of AZD3147, a new highly selective inhibitor of TORC1 and TORC2 (Pike et al., 2015) that can circumvent the discontinuity of clinical trials (Martelli et al., 2018). Similarly, other derived compounds were developed in parallel like WYE-125132 (WYE-132) or Torin2 (Liu et al., 2013; Yu et al., 2010). WYE-132 has better pharmacokinetics properties than WYE-354 and is highly selective for mTOR over PI3K and the PIKK ATR (Yu et al., 2010). Torin2 has improved pharmacological and solubility properties compared to its structural analogue Torin1 but also significant activity against mTOR, ATM, ATR, and DNA-PK, as well as both in vitro and in vivo antitumor efficacy, being therefore a potent broadly active pan-PIKK kinase inhibitor (Liu et al., 2013). Indeed, if Torin2 most potently inhibits mTORC1 and mTORC2 in vivo at concentrations of less than $10 \mathrm{nM}$, it also inhibits ATR, ATM, and DNA-PK at concentrations between 20 and $100 \mathrm{nM}$ and PI3K at concentrations above 200 $\mathrm{nM}$. This is in contrast with Torin1 which only exhibits moderate inhibition of DNA-PK (250 nM) but is inactive against other PIKK-family kinases. Unexpectedly, Torin2 also has a lower residence time than Torin1 (4h vs 16h) leading to suggest that Torins might induce a TOR conformation change in the kinase that is energetically difficult to recover from rather than different binding affinities. This adds a specific feature to Torins that distinguish them from other asTORis, showing that Torin 1 and Torin2 although structurally close cannot only be compared in terms of potency. Moreover, the intentional development of new dual Torin2 analogs that inhibit both mTOR and ATR (Shaik et al., 2018) for clinical purposes shows that driving drug selectivity to specificity is a difficult chemistry task. Therefore, the crosstalk between TOR pathway and other pathways such as the DNA damage response (Li et al., 2012; Silvera et al., 2017) involving other PIKK close to TOR might hamper the discovery 
of true TOR targets with Torin 2 and Torin2 analogs. In addition, Torin 2 was reported as an antimalarial agent 1,000-fold selective to malaria parasites over mammalian cells whereas TOR homolog is not found in Plasmodium falciparum (Hanson et al., 2013). Therefore, Torin2 is particularly known to target other eukaryotic proteins than TOR and should be used with caution for biological studies.

Nowadays, the use of more than one selective and potent inhibitor through targeting for instance two pathways or sub-pathways is more and more explored in medicine rather than using dual $\mathrm{PI} 3 \mathrm{~K} / \mathrm{mTOR}$ inhibitors of a single pathway, which can have the "possible drawback of association with greater toxicity" (Simioni et al., 2014). Furthermore, novel compounds are continuously searched to circumvent the discontinuity of potent and selective compounds yet cytostatic or unstable in clinical trials (Andrs et al., 2015; Chen et al., 2012; Estrada et al., 2013; Fraser et al., 2016; Mortensen et al., 2015; Nowak et al., 2009; Park et al., 2014; Pei et al., 2012; Slotkin et al., 2015; Walters and Cox, 2018; Zheng and Jiang, 2015). Thus, although selectivity is not always the major criteria in clinical trials, it is an essential criteria for the choice of an inhibitor to elucidate the role of a particular kinase in biological tissues and in vitro studies (Arrowsmith et al., 2015). This illustrates why potency and efficacy of selective inhibitors should be carefully examined and that the concept of inhibitor strength can be misleading according to experts (Michel and Seifert, 2015). This also underlines that testing/using more than one TOR ATP competitive inhibitor should help identifying and confirming TOR-dependent regulated functions.

\section{ATP-competitive TOR inhibitors in plant and algae}

The first asTORis that have been used in plants were among those presented above, i.e., KU-0063794, AZD-8055, Torin1 and Torin2, WYE-354 and WYE-132. It was remarkable that KU-0063794 and WYE-354 and their improved derived molecules, AZD-8055 and WYE-132, followed the same relative potency than in mammalian cells, i.e., AZD-8055 > KU-0063794 and WYE-132 > WYE354 
(Table 1) (Montane and Menand, 2013). We encountered solubility problems with Torin1 in our culture conditions, and among the drugs we could get and test at that time; AZD-8055 (Fig. 1D) and WYE-132 quickly became our favorites to fully inhibit Arabidopsis WT seedlings growth because of high reproducibility of responses and of temporal stability of dose-dependent inhibition. Furthermore, we demonstrated that the plant growth inhibition by AZD-8055 and WYE-132 is TOR-dependent by showing induced haploinsufficiency to TOR as the TOR/tor heterozygote mutants are hypersensitive to both inhibitors compared to WT (Fig. 1D) (Montane and Menand, 2013). Maximal efficacy was shown through dose-response curves showing complete inhibition of root growth, as opposed to rapamycin in plants or algae (Fig. 1C-F). asTORis were potent in most photosynthetic eukaryotes as they strongly inhibit growth of a large variety of plants (Arabidopsis, potato, tomato, rice; Lotus, millet, Nicotiana), and proliferation in both green algae and diatoms (C. reinhardtii, $P$. tricornutum) (Deng et al., 2017; Dong et al., 2015; Dong et al., 2018; Imamura et al., 2016; Montane and Menand, 2013; Prioretti et al., 2017; Song et al., 2017). Table 1 and Fig. 2 show the concentration range of the main asTORis used to inhibit proliferation, to study TOR functions and that helped find new TOR targets in photosynthetic eukaryotes: KU-0063794, AZD-8055, Torin1 and Torin2 (Deng et al., 2017; Dong et al., 2015; Kravchenko et al., 2015; Li et al., 2015; Li et al., 2017; Mohammed et al., 2018; Montane and Menand, 2013; Mubeen et al., 2018; Ouibrahim et al., 2015; Pfeiffer et al., 2016; Prioretti et al., 2017; Schepetilnikov et al., 2013; Schepetilnikov et al., 2011; Schepetilnikov et al., 2017; Wang et al., 2018b; Werth et al., 2018). Some authors tried to establish a scale of "strength" of these inhibitors in plants starting from values of IC50 kinase in vitro (Deng et al., 2016; Xiong et al., 2017a). Here, as we have discussed above, we would like to stress that knowledge from the biochemical and animal fields should be taken in consideration when using such inhibitors. Indeed, as shown above for KU-0063794, Torin1 and AZD-8055 (Table 1), using the IC50 of in vitro kinase to predict IC50 of proliferation hardly works in mammals and plants. This might hide other characteristics of a drug in vivo as well as its speciation in culture medium. As reported above, the 
potency of Torin 1 in animal cells was higher than that of KU-0063794 but the selectivity was the opposite. Regarding the use of Torins and in particular Torin2 as an asTORi, we have mentioned above that it was described as a pan-PIKK family inhibitor as it exhibited potent biochemical and cellular activity against PIKKs, including ATM and ATR (Liu et al., 2013). Indeed, mammalian mTOR is involved in the network of responses between DNA damage and cell cycle control, including the activity of the ATM/ATR-CHK1/CHK2-p53 axis (Silvera et al., 2017). Since ATM and ATR are conserved in plants and algae and regarding the known role of Arabidopsis ATM in regulation of meristem activity and DNA-damage responses (Fulcher and Sablowski, 2009; Hisanaga et al., 2013; Ricaud et al., 2007), care should be taken when analyzing data with Torin2 in plants, especially when high concentrations are used as mentioned above. For our part, we therefore avoid using Torins in order to selectively inhibit the TOR pathway and checked that selective ATP competitive ATM inhibitors did not inhibit growth and so could be used as a selectivity control toward TOR inhibitors (Montane and Menand, 2013). The specificity of a cellular response towards TOR inhibition can also be confirmed in plant and algae through comparing the effect of different selective asTORis such as AZD-8055 and WYE-132 (Barrada et al., 2019; Prioretti et al., 2017) (Prioretti et al., 2017)but it has to be done at doses leading to similar growth inhibition. Furthermore, we should keep in mind that we should not expect identical molecular and cellular effects of rapamycin and asTORis in plants and algae as rapamycin does not inhibit all TORC1 activity, and might also not inhibit other potential new plant/algae TOR complexes (see above). Taking into account the conservation of the minimal core TORC1 in plants, and the specificity of plant targets that only start to emerge, we think that other plant TOR target sites than the sole S6K T449 will need to be developed to accurately record the level of plant TOR inhibition. Indeed, the mammalian S6K1 T389 equivalent of plant S6K T449 corresponds to a poor TOR target (Kang et al., 2013). 

for genetic screens. First, they allow avoiding possible drawbacks associated with transgenic lines. Second, they allow to control the time of and the level of inhibition on a given organism, organ, tissue or cell by following the kinetics of any measurable parameter and its dose-dependent evolution, an important aspect in case of essential genes like TOR. Inhibiting TOR from a known WT physiological context is definitely different than comparing loss of function or overexpressing functions. Furthermore, being cytostatic also in plants (Montane and Menand, 2013), these inhibitors offer the possibility to rescue hypersensitive plants and to more accurately follow reciprocal changes or point out any differential behavior of targets when inhibition is relaxed. To date, few screens of mutants resistant or hypersensitive to TOR inhibitors have been reported in plants and algae yet they revealed important aspect of TOR signaling (Barrada et al., 2019; Couso et al., 2016; Crespo et al., 2005; Li et al., 2015). Recently, the vip1-1 C. reinhardtii mutant hypersensitive to the first saturating concentration of $500 \mathrm{nM}$ rapamycin inhibiting growth to the $50 \%$ plateau value was isolated (Couso et al., 2016). This mutant which was also hypersensitive to the single concentration tested of $500 \mathrm{nM}$ AZD-8055 or Torin1 revealed an interaction between TOR and inositol polyphosphate intracellular signaling. In Arabidopsis, a first screen of mutants that show no chlorosis of cotyledons induced by 2 $\mu \mathrm{M}$ AZD-8055 allowed to select 9 mutants among which a new ABI4 (ABA-INSENSITIVE 4) allele, revealing a new role of TOR in abscisic acid (ABA) signaling (Li et al., 2015). Interestingly, in our culture conditions we never observed AZD-8055-induced chlorosis at doses up to $10 \mu \mathrm{M}$ (Montane and Menand, 2013), meaning that growth conditions have to be considered consistently with the role of TOR in response to nutrients and stress. We recently screened EMS Arabidopsis mutants resistant to a concentration of $1 \mu \mathrm{M}$ AZD-8055 inhibiting $90 \%$ of root lengthening and discovered that the homolog of yeast YAK1 (Yet Another Kinase 1) and human DYRK1A (Dual Specificity Tyrosine 
plant cell proliferation (Barrada et al., 2019). We also showed that pINDY, an-ATP competitive inhibitor of DYRK1A, mimics Arabidopsis yakl loss-of-function mutations. This offers a new way to study TOR-YAK1 axis in plants. In addition, we isolated other mutants sensitive or resistant to AZD8055 concentrations leading to other levels of growth inhibition which are now under study. Hence, together with studies of known mutants of components of the plant/algae TORC1 pathway, new screening approaches might actively help deciphering TOR pathways in photosynthetic organisms.

In addition to being proof-of-concept that the pharmacogenetic screens can help identify new functions of TOR in plant and algae, these studies remind the importance to carefully design the conditions of the screen but also of further studies. For instance, the haploinsufficiency phenotype of TOR/tor heterozygotes discussed above is a nice illustration of the importance of the "right" dose of an inhibitor to compare physiological context of two genetic backgrounds (Montane and Menand, 2013). The TOR/tor heterozygotes were clearly hypersensitive to AZD-8055 concentrations between 0.1 and $1 \mu \mathrm{M}$, but grow the same as the WT at doses below $0.03 \mu \mathrm{M}$ and above the maximal inhibitory concentrations of $3 \mu \mathrm{M}$, that almost completely inhibit root growth (Fig. 1 D). For instance, to compare YAK1- and TOR- expression patterns by GUS staining in roots which growth was similarly inhibited by AZD-8055, we used twice lower AZD-8055 concentration for the GUS knock-in TOR/tor-1 heterozygous line (Menand et al., 2002) than for a $p Y A K 1:: Y A K 1-G U S$ homozygous line (Barrada et al., 2019). Therefore, the dose-dependent effect of asTORis on the processes analyzed should be preliminary determined prior to design specific genetic screen. We would like to finish this section with guidelines that might help plan experiments with TOR inhibitors in plants and algae, with an emphasis on pharmacogenetic screening.

Guidelines for plant physiology studies and genetic screens with asTORis:

- Clarify the question you want to answer to choose the best developmental or growth stage of the plant or algae to study or to screen, 
593

594

595

596

597

598

599

600

601

602

603

604

605

606

607

608

609

610

611 - design peptide(s) encompassing the putative phosphorylation site(s) to demonstrate which one is

612 TOR-dependent,

613 - express the WT and mutated form (s) in phosphorylated amino acid of the new target in a knock614 out mutant and compare dose response curves.

615

\section{Conclusion}


617

618

619

620

621

622

623

624

625

626

627

628

629

630

631

632

633

634

635

636

637

638

639

640

641

If rapamycin has opened the study of TOR functions in plant and algae as in yeast and mammals, it should be used cautiously in plants for which overexpression of FKBP12 is required. The use of rapamycin has fewer drawbacks in some algae, like Chlamydomonas reinhardtii, which is naturally sensitive to rapamycin. However, its good potency masks incomplete efficacy in many if not all species studied till now. The requirement of FKBP12 partner to inhibit TOR with rapamycin might also disturb signaling responses due to the cellular role of FKBP12 and putative off targets of rapamycin itself might also interfere. However, in any case, we should keep in mind that rapamycin does not inhibit all TORC1 activity and does not inhibit other TOR complexes potentially present in plants and algae. Conversely, ATP-competitive TOR inhibitors more efficiently inhibit proliferation and growth than rapamycin in algae and plants, as in animals, and are therefore very good tools to study the TOR pathway in photosynthetic eukaryotes. However, we should in return take care of information's about concentration range and singularity from chemical and animal researchers who developed and experienced them. Their use has already helped decipher TOR pathway effectors in plants and algae and we guess they will certainly be of great help in the future.

\section{Supplementary data}

Figure S1

\section{Acknowledgements}

Work on TOR in our laboratory was supported by Agence Nationale de la Recherche (ANR) grants SIGNAUXBioNRJ (ANR-15-CE05-0021-03), TRANSLATOR (ANR-11-BSV6-0010) and DECORATORS (ANR-14-CE19-0007). We apologize to our colleagues whose work could not be included due to space limitations. No conflict of interest declared. 


\section{References}

643 Aagaard-Tillery K. M., Jelinek D. F. 1994. Inhibition of Human B-Lymphocyte Cell-Cycle Progression and 644 Differentiation by Rapamycin. Cellular Immunology 156, 493-507.

645 Aghdasi B., Ye K., Resnick A., Huang A., Ha H. C., Guo X., Dawson T. M., Dawson V. L., Snyder S. H. 2001. 646 FKBP12, the 12-kDa FK506-binding protein, is a physiologic regulator of the cell cycle. Proceedings of the 647 National Academy of Sciences, USA 98, 2425-2430.

648 Ahn C. S., Han J. A., Lee H. S., Lee S., Pai H. S. 2011. The PP2A regulatory subunit Tap46, a component of the 649 TOR signaling pathway, modulates growth and metabolism in plants. The Plant Cell 23, 185-209.

650 Alavilli H., Lee H., Park M., Yun D. J., Lee B. H. 2018. Enhanced multiple stress tolerance in Arabidopsis by 651 overexpression of the polar moss peptidyl prolyl isomerase FKBP12 gene. Plant Cell Reports 37, 453-465.

652 Alessi D. R., Pearce L. R., Garcia-Martinez J. M. 2009. New Insights into mTOR Signaling: mTORC2 and Beyond. 653 Science Signaling 2.

654 Anderson G. H., Veit B., Hanson M. R. 2005. The Arabidopsis AtRaptor genes are essential for post-embryonic plant growth. BMC Biology 3, 12.

Andrs M., Korabecny J., Jun D., Hodny Z., Bartek J., Kuca K. 2015. Phosphatidylinositol 3-Kinase (PI3K) and phosphatidylinositol 3-kinase-related kinase (PIKK) inhibitors: importance of the morpholine ring. Journal of Medical Chemistry 58, 41-71.

Arrowsmith C. H., Audia J. E., Austin C., et al. 2015. The promise and peril of chemical probes. Nature Chemical Biology 11, 536-541.

Barbet N. C., Schneider U., Helliwell S. B., Stansfield I., Tuite M. F., Hall M. N. 1996. TOR controls translation initiation and early G1 progression in yeast. Molecular Biology of the Cell 7, 25-42.

Barrada A., Djendli M., Desnos T., Mercier R., Robaglia C., Montane M. H., Menand B. 2019. A TOR-YAK1 signaling axis controls cell cycle, meristem activity and plant growth in Arabidopsis. Development doi:10.1242/dev.171298.

Bassham D. C. 2009. Function and regulation of macroautophagy in plants. Biochimica et Biophysica Acta 1793, 1397-1403.

Batool A., Aashaq S., Andrabi K. I. 2017. Reappraisal to the study of 4E-BP1 as an mTOR substrate - A normative critique. European Journal of Cell Biology 96, 325-336.

Bello T., Gujral T. S. 2018. KInhibition: A Kinase Inhibitor Selection Portal. iScience 8, 49-53.

Ben-Sahra I., Manning B. D. 2017. mTORC1 signaling and the metabolic control of cell growth. Current Opinion in Cell Biology 45, 72-82.

Benjamin D., Colombi M., Moroni C., Hall M. N. 2011. Rapamycin passes the torch: a new generation of mTOR inhibitors. Nature Reviews Drug Discovery 10, 868-880.

Betz C., Hall M. N. 2013. Where is mTOR and what is it doing there? Journal of Cell Biology 203, 563-574.

Blagosklonny M. V. 2012. Cell cycle arrest is not yet senescence, which is not just cell cycle arrest: terminology for TOR-driven aging. Aging (Albany NY) 4, 159-165.

Caldana C., Li Y., Leisse A., Zhang Y., Bartholomaeus L., Fernie A. R., Willmitzer L., Giavalisco P. 2013. Systemic analysis of inducible target of rapamycin mutants reveal a general metabolic switch controlling growth in Arabidopsis thaliana. The Plant Journal 73, 897-909.

Carlson C. B., Robers M. B., Vogel K. W., Machleidt T. 2009. Development of LanthaScreen cellular assays for key components within the PI3K/AKT/mTOR pathway. Journal of Biomolecular Screening 14, 121-132.

Chen S. M., Liu J. L., Wang X., Liang C., Ding J., Meng L. H. 2012. Inhibition of tumor cell growth, proliferation and migration by X-387, a novel active-site in hibitor of mTOR. Biochemistry and Pharmacology 83, 1183-1194.

Choi J., Chen J., Schreiber S. L., Clardy J. 1996. Structure of the FKBP12-rapamycin complex interacting with the binding domain of human FRAP. Science 273, 239-242.

Chresta C. M., Davies B. R., Hickson I., et al. 2012. AZD8055 is a potent, selective, and orally bioavailable ATPcompetitive mammalian target of rapamycin kinase inhibitor with in vitro and in vivo antitumor activity. Cancer Research 70, 288-298. 
Couso I., Evans B. S., Li J., Liu Y., Ma F., Diamond S., Allen D. K., Umen J. G. 2016. Synergism between Inositol Polyphosphates and TOR Kinase Signaling in Nutrient Sensing, Growth Control, and Lipid Metabolism in Chlamydomonas. The Plant Cell 28, 2026-2042.

Crespo J. L., Diaz-Troya S., Florencio F. J. 2005. Inhibition of target of rapamycin signaling by rapamycin in the unicellular green alga Chlamydomonas reinhardtii. Plant Physiololgy 139, 1736-1749.

Cunningham J. T., Rodgers J. T., Arlow D. H., Vazquez F., Mootha V. K., Puigserver P. 2007. mTOR controls mitochondrial oxidative function through a YY1-PGC-1alpha transcriptional complex. Nature 450, 736-740.

Davis M. I., Hunt J. P., Herrgard S., Ciceri P., Wodicka L. M., Pallares G., Hocker M., Treiber D. K., Zarrinkar P. P. 2011. Comprehensive analysis of kinase inhibitor selectivity. Nature Biotechnology 29, 1046-1051.

De Cicco M., Rahim M. S., Dames S. A. 2015. Regulation of the Target of Rapamycin and Other Phosphatidylinositol 3-Kinase-Related Kinases by Membrane Targeting. Membranes (Basel) 5, 553-575.

Deng K., Dong P., Wang W., et al. 2017. The TOR Pathway Is Involved in Adventitious Root Formation in Arabidopsis and Potato. Frontiers in Plant Science 8, 784.

Deng K., Yu L., Zheng X., Zhang K., Wang W., Dong P., Zhang J., Ren M. 2016. Target of Rapamycin Is a Key Player for Auxin Signaling Transduction in Arabidopsis. Frontiers in Plant Science 7, 291.

Deprost D., Truong H. N., Robaglia C., Meyer C. 2005. An Arabidopsis homolog of RAPTOR/KOG1 is essential for early embryo development. Biochemical and Biophysical Research Communications 326, 844-850.

Dobrenel T., Caldana C., Hanson J., Robaglia C., Vincentz M., Veit B., Meyer C. 2016a. TOR Signaling and Nutrient Sensing. Annual Review of Plant Biology 67, 261-285.

Dobrenel T., Mancera-Martinez E., Forzani C., et al. 2016b. The Arabidopsis TOR Kinase Specifically Regulates the Expression of Nuclear Genes Coding for Plastidic Ribosomal Proteins and the Phosphorylation of the Cytosolic Ribosomal Protein S6. Frontiers in Plant Science 7, 1611.

Dong P., Xiong F., Que Y., Wang K., Yu L., Li Z., Ren M. 2015. Expression profiling and functional analysis reveals that TOR is a key player in regulating photosynthesis and phytohormone signaling pathways in Arabidopsis. Frontiers in Plant Science 6, 677.

Dong Q., Mao K., Duan D., et al. 2018. Genome-wide analyses of genes encoding FK506-binding proteins reveal their involvement in abiotic stress responses in apple. BMC Genomics 19, 707.

Dowling R. J., Topisirovic I., Alain T., et al. 2010a. mTORC1-mediated cell proliferation, but not cell growth, controlled by the 4E-BPs. Science 328, 1172-1176.

Dowling R. J., Topisirovic I., Fonseca B. D., Sonenberg N. 2010b. Dissecting the role of mTOR: lessons from mTOR inhibitors. Biochimica et Biophysica Acta 1804, 433-439.

Dunlop E. A., Tee A. R. 2013. The kinase triad, AMPK, mTORC1 and ULK1, maintains energy and nutrient homoeostasis. Biochemical Society Transactions 41, 939-943.

Duvel K., Yecies J. L., Menon S., et al. 2010. Activation of a Metabolic Gene Regulatory Network Downstream of mTOR Complex 1. Molecular Cell 39, 171-183.

Eltschinger S., Loewith R. 2016. TOR Complexes and the Maintenance of Cellular Homeostasis. Trends in Cell Biology 26, 148-159.

Estrada A. A., Shore D. G., Blackwood E., et al. 2013. Pyrimidoaminotropanes as potent, selective, and efficacious small molecule kinase inhibitors of the mammalian target of rapamycin (mTOR). Journal of Medical Chemistry 56, 3090-3101.

Feldman M. E., Apsel B., Uotila A., Loewith R., Knight Z. A., Ruggero D., Shokat K. M. 2009. Active-site inhibitors of mTOR target rapamycin-resistant outputs of mTORC1 and mTORC2. PLoS Biology 7, e38.

Fraser C., Carragher N. O., Unciti-Broceta A. 2016. ECF309: a potent, selective and cell-permeable mTOR inhibitor. MedChemComm 7, 471-477.

Fulcher N., Sablowski R. 2009. Hypersensitivity to DNA damage in plant stem cell niches. Proceedings of the National Academy of Sciences, USA 106, 20984-20988.

Garcia-Echeverria C. 2011. Blocking the mTOR pathway: a drug discovery perspective. Biochemical Society Transactions 39, 451-455.

Garcia-Martinez J. M., Moran J., Clarke R. G., Gray A., Cosulich S. C., Chresta C. M., Alessi D. R. 2009. Ku0063794 is a specific inhibitor of the mammalian target of rapamycin (mTOR). Biochemical Journal 421, $29-42$. 
Gaubitz C., Prouteau M., Kusmider B., Loewith R. 2016. TORC2 Structure and Function. Trends in Biochemcal Sciences 41, 532-545.

Geisler M., Bailly A. 2007. Tete-a-tete: the function of FKBPs in plant development. Trends in Plant Science 12, 465-473.

Giubellino A., Bullova P., Nolting E., et al. 2013. Combined Inhibition of mTORC1 and mTORC2 Signaling Pathways Is a Promising Therapeutic Option in Inhibiting Pheochromocytoma Tumor Growth: In Vitro and In Vivo Studies in Female Athymic Nude Mice. Endocrinology 154, 646-655.

Gold L. I., Sung J. J., Siebert J. W., Longaker M. T. 1997. Type I (RI) and type II (RII) receptors for transforming growth factor-beta isoforms are expressed subsequent to transforming growth factor-beta ligands during excisional wound repair. American Journal of Pathology 150, 209-222.

Gonzalez S., Rallis C. 2017. The TOR Signaling Pathway in Spatial and Temporal Control of Cell Size and Growth. Frontiers in Cell and Developmental Biology 5, 61.

Guertin D. A., Sabatini D. M. 2007. Defining the role of mTOR in cancer. Cancer Cell 12, 9-22.

Guertin D. A., Sabatini D. M. 2009. The pharmacology of mTOR inhibition. Science Signaling 2, pe24.

Hall M. N. 2016. TOR and paradigm change: cell growth is controlled. Molecular Biology of the Cell 27, 28042806.

Hanson K. K., Ressurreicao A. S., Buchholz K., et al. 2013. Torins are potent antimalarials that block replenishment of Plasmodium liver stage parasitophorous vacuole membrane proteins. Proceedings of the National Academy of Sciences, USA 110, E2838-E2847.

Harwood F. C., Klein Geltink R. I., O'Hara B. P., et al. 2018. ETV7 is an essential component of a rapamycininsensitive mTOR complex in cancer. Science Advances 4, eaar3938.

Heitman J., Movva N. R., Hall M. N. 1991. Targets for cell cycle arrest by the immunosuppressant rapamycin in yeast. Science 253, 905-909.

Henriques R., Magyar Z., Monardes A., et al. 2010. Arabidopsis S6 kinase mutants display chromosome instability and altered RBR1-E2F pathway activity. EMBO Journal 29, 2979-2993.

Hisanaga T., Ferjani A., Horiguchi G., et al. 2013. The ATM-dependent DNA damage response acts as an upstream trigger for compensation in the fas 1 mutation during Arabidopsis leaf development. Plant Physiology $162,831-841$.

Huang S. L., Bjornsti M. A., Houghton P. J. 2003. Rapamycins - Mechanism of action and cellular resistance. Cancer Biology \& Therapy 2, 222-232.

Huggins D. J., Sherman W., Tidor B. 2012. Rational approaches to improving selectivity in drug design. Journal of Medicinal Chemistry 55, 1424-1444.

Imamura S., Ishiwata A., Watanabe S., Yoshikawa H., Tanaka K. 2013. Expression of budding yeast FKBP12 confers rapamycin susceptibility to the unicellular red alga Cyanidioschyzon merolae. Biochemical and Biophysical Research Communications 439, 264-269.

Imamura S., Kawase Y., Kobayashi I., Shimojima M., Ohta H., Tanaka K. 2016. TOR (target of rapamycin) is a key regulator of triacylglycerol accumulation in microalgae. Plant Signaling \& Behavior 11, e1149285.

Jhanwar-Uniyal M., Amin A. G., Cooper J. B., Das K., Schmidt M. H., Murali R. 2017. Discrete signaling mechanisms of mTORC1 and mTORC2: Connected yet apart in cellular and molecular aspects. Advances in Biological Regulation 64, 39-48.

Juppner J., Mubeen U., Leisse A., Caldana C., Wiszniewski A., Steinhauser D., Giavalisco P. 2018. The target of rapamycin kinase affects biomass accumulation and cell cycle progression by altering carbon/nitrogen balance in synchronized Chlamydomonas reinhardtii cells. The Plant Journal 93, 355-376.

Kamada Y., Yoshino K., Kondo C., Kawamata T., Oshiro N., Yonezawa K., Ohsumi Y. 2010. Tor Directly Controls the Atg1 Kinase Complex To Regulate Autophagy. Molecular and Cellular Biology 30, 1049-1058.

Kang S. A., Pacold M. E., Cervantes C. L., et al. 2013. mTORC1 phosphorylation sites encode their sensitivity to starvation and rapamycin. Science 341, 1236566.

Karaman M. W., Herrgard S., Treiber D. K., et al. 2008. A quantitative analysis of kinase inhibitor selectivity. Nature Biotechnology 26, 127-132.

Kazyken D., Kaz Y., Kiyan V., Zhylkibayev A. A., Chen C. H., Agarwal N. K., Sarbassov dos D. 2014. The nuclear import of ribosomal proteins is regulated by mTOR. Oncotarget 5, 9577-9593. 
Kim J., Kundu M., Viollet B., Guan K. L. 2011. AMPK and mTOR regulate autophagy through direct phosphorylation of Ulk1. Nature Cell Biology 13, 132-U171.

Kravchenko A., Citerne S., Jehanno I., Bersimbaev R. I., Veit B., Meyer C., Leprince A. S. 2015. Mutations in the Arabidopsis Lst8 and Raptor genes encoding partners of the TOR complex, or inhibition of TOR activity decrease abscisic acid (ABA) synthesis. Biochemical and Biophysical Research Communications 467, 992-997. Leiber R. M., John F., Verhertbruggen Y., Diet A., Knox J. P., Ringli C. 2010. The TOR pathway modulates the structure of cell walls in Arabidopsis. The Plant Cell 22, 1898-1908.

Li F. Q., Vierstra R. D. 2014. Arabidopsis ATG11, a scaffold that links the ATG1-ATG13 kinase complex to general autophagy and selective mitophagy. Autophagy 10, 1466-1467.

Li L., Song Y., Wang K., Dong P., Zhang X., Li F., Li Z., Ren M. 2015. TOR-inhibitor insensitive-1 (TRIN1) regulates cotyledons greening in Arabidopsis. Frontiers in Plant Science 6, 861.

Li X., Cai W., Liu Y., et al. 2017. Differential TOR activation and cell proliferation in Arabidopsis root and shoot apexes. Proceedings of the National Academy of Sciences, USA 114, 2765-2770.

Li Y., Mitsuhashi S., Ikejo M., Miura N., Kawamura T., Hamakubo T., Ubukata M. 2012. Relationship between ATM and ribosomal protein S6 revealed by the chemical inhibition of Ser/Thr protein phosphatase type 1. Bioscience, Biotechnology, and Biochemistry 76, 486-494.

Liu Q., Chang J. W., Wang J., et al. 2010. Discovery of 1-(4-(4-propionylpiperazin-1-yl)-3(trifluoromethyl)phenyl)-9-(quinolin-3-yl)benz o[h][1,6]naphthyridin-2(1H)-one as a highly potent, selective mammalian target of rapamycin (mTOR) inhibitor for the treatment of cancer. Journal of Medicinal Chemistry 53, 7146-7155.

Liu Q., Kirubakaran S., Hur W., et al. 2012a. Kinome-wide selectivity profiling of ATP-competitive mammalian target of rapamycin (mTOR) inhibitors and characterization of their binding kinetics. Journal of Biological Chemistry 287, 9742-9752.

Liu Q., Ren T., Fresques T., et al. 2012b. Selective ATP-competitive inhibitors of TOR suppress rapamycininsensitive function of TORC2 in Saccharomyces cerevisiae. ACS Chemical Biology 7, 982-987.

Liu Q., Xu C., Kirubakaran S., et al. 2013. Characterization of Torin2, an ATP-competitive inhibitor of mTOR, ATM, and ATR. Cancer Research 73, 2574-2586.

Liu Y. M., Bassham D. C. 2010. TOR Is a Negative Regulator of Autophagy in Arabidopsis thaliana. Plos One 5.

Luo Y., Liu L., Wu Y., Singh K., Su B., Zhang N., Liu X., Shen Y., Huang S. 2015. Rapamycin inhibits mSin1 phosphorylation independently of MTORC1 and mTORC2. Oncotarget 6, 4286-4298.

Magnuson B., Ekim B., Fingar D. C. 2012. Regulation and function of ribosomal protein S6 kinase (S6K) within mTOR signalling networks. Biochemical Journal 441, 1-21.

Mahfouz M. M., Kim S., Delauney A. J., Verma D. P. 2006. Arabidopsis TARGET OF RAPAMYCIN interacts with RAPTOR, which regulates the activity of S6 kinase in response to osmotic stress signals. The Plant Cell 18, $477-$ 490.

Marshall G., Howard Z., Dry J., et al. 2011. Benefits of mTOR kinase targeting in oncology: pre-clinical evidence with AZD8055. Biochemical Society Transactions 39, 456-459.

Marshall R. S., Li F. Q., Gemperline D. C., Book A. J., Vierstra R. D. 2015. Autophagic Degradation of the 26S Proteasome Is Mediated by the Dual ATG8/Ubiquitin Receptor RPN10 in Arabidopsis. Molecular Cell 58, 10531066.

Martelli A. M., Buontempo F., McCubrey J. A. 2018. Drug discovery targeting the mTOR pathway. Clinical Science 132, 543-568.

Masclaux-Daubresse C., Chen Q. W., Have M. 2017. Regulation of nutrient recycling via autophagy. Current Opinion in Plant Biology 39, 8-17.

Menand B., Desnos T., Nussaume L., Berger F., Bouchez D., Meyer C., Robaglia C. 2002. Expression and disruption of the Arabidopsis TOR (target of rapamycin) gene. Proceedings of the National Academy of Sciences, USA 99, 6422-6427.

Meyuhas 0. 2015. Ribosomal Protein S6 Phosphorylation: Four Decades of Research. International Review of Cell and Molecular Biology 320, 41-73.

Meyuhas O., Dreazen A. 2009. Ribosomal protein S6 kinase from TOP mRNAs to cell size. Progress in Molecular Biology and Translational Science 90, 109-153. 
Michel M. C., Seifert R. 2015. Selectivity of pharmacological tools: implications for use in cell physiology. A review in the theme: Cell signaling: proteins, pathways and mechanisms. American Journal of Physiology-Cell Physiology 308, C505-520.

Mohammed B., Bilooei S. F., Doczi R., Grove E., Railo S., Palme K., Ditengou F. A., Bogre L., Lopez-Juez E. 2018. Converging Light, Energy and Hormonal Signaling Control Meristem Activity, Leaf Initiation, and Growth. Plant Physiology 176, 1365-1381.

Montane M. H., Menand B. 2013. ATP-competitive mTOR kinase inhibitors delay plant growth by triggering early differentiation of meristematic cells but no developmental patterning change. Journal of Experimental Botany 64, 4361-4374.

Moreau M., Azzopardi M., Clement G., et al. 2012. Mutations in the Arabidopsis homolog of LST8/GbetaL, a partner of the target of Rapamycin kinase, impair plant growth, flowering, and metabolic adaptation to long days. The Plant Cell 24, 463-481.

Mortensen D. S., Perrin-Ninkovic S. M., Shevlin G., et al. 2015. Discovery of mammalian target of rapamycin (mTOR) kinase inhibitor CC-223. Journal of Medicinal Chemistry 58, 5323-5333.

Mosby's Medical Dictionary. 2009. Retrieved October $31 \quad 2018$ from https://medicaldictionary.thefreedictionary.com/drug+potency.

Mubeen U., Juppner J., Alpers J., Hincha D. K., Giavalisco P. 2018. Target of Rapamycin Inhibition in Chlamydomonas reinhardtii Triggers de-novo Amino Acid Synthesis by Enhancing Nitrogen Assimilation. The Plant Cell.

Mukaida S., Ogawa T., Ohishi K., Tanizawa Y., Ohta D., Arita M. 2016. The effect of rapamycin on biodieselproducing protist Euglena gracilis. Bioscience Biotechnology and Biochemistry 80, 1223-1229.

Mukhopadhyay S., Frias M. A., Chatterjee A., Yellen P., Foster D. A. 2016. The Enigma of Rapamycin Dosage. Molecular Cancer Therapeutics 15, 347-353.

Nazio F., Strappazzon F., Antonioli M., et al. 2013. mTOR inhibits autophagy by controlling ULK1 ubiquitylation, self-association and function through AMBRA1 and TRAF6. Nature Cell Biology 15, 406-416.

Nowak P., Cole D. C., Brooijmans N., et al. 2009. Discovery of potent and selective inhibitors of the mammalian target of rapamycin (mTOR) kinase. Journal of Medicinal Chemistry 52, 7081-7089.

Osmulski P. A., Gaczynska M. 2013. Rapamycin allosterically inhibits the proteasome. Molecular Pharmacology 84, 104-113.

Ouibrahim L., Rubio A. G., Moretti A., Montane M. H., Menand B., Meyer C., Robaglia C., Caranta C. 2015. Potyviruses differ in their requirement for TOR signalling. Journal of General Virology 96, 2898-2903.

Pancha I., Shima H., Higashitani N., Igarashi K., Higashitani A., Tanaka K., Imamura S. 2018. Target of rapamycin-signaling modulates starch accumulation via glycogenin phosphorylation status in the unicellular red alga Cyanidioschyzon merolae. The Plant Journal, doi.org/10.1111/tpj.14136.

Park H., Choe H., Hong S. 2014. Virtual screening and biochemical evaluation to identify new inhibitors of mammalian target of rapamycin (mTOR). Bioorganic \& Medicinal Chemistry Letters 24, 835-838.

Patrick R. M., Lee J. C. H., Teetsel J. R. J., Yang S. H., Choy G. S., Browning K. S. 2018. Discovery and characterization of conserved binding of elF4E 1 (CBE1), a eukaryotic translation initiation factor 4E-binding plant protein. Journal of Biological Chemistry 293, 17240-17247.

Pei Z., Blackwood E., Liu L., et al. 2012. Discovery and Biological Profiling of Potent and Selective mTOR Inhibitor GDC-0349. ACS Medicinal Chemistry Letters 4, 103-107.

Peng T., Golub T. R., Sabatini D. M. 2002. The immuno suppress ant rapamycin mimics a starvation-like signal distinct from amino acid and glucose deprivation. Molecular and Cellular Biology 22, 5575-5584.

Perez-Perez M. E., Couso I., Crespo J. L. 2017. The TOR Signaling Network in the Model Unicellular Green Alga Chlamydomonas reinhardtii. Biomolecules 7.

Perez-Perez M. E., Florencio F. J., Crespo J. L. 2010. Inhibition of Target of Rapamycin Signaling and Stress Activate Autophagy in Chlamydomonas reinhardtii. Plant Physiology 152, 1874-1888.

Pfeiffer A., Janocha D., Dong Y., et al. 2016. Integration of light and metabolic signals for stem cell activation at the shoot apical meristem. Elife 5. 
Philippe L., Vasseur J. J., Debart F., Thoreen C. C. 2018. La-related protein 1 (LARP1) repression of TOP mRNA translation is mediated through its cap-binding domain and controlled by an adjacent regulatory region. Nucleic Acids Research 46, 1457-1469.

Pike K. G., Malagu K., Hummersone M. G., et al. 2013. Optimization of potent and selective dual mTORC1 and mTORC2 inhibitors: the discovery of AZD8055 and AZD2014. Bioorganic \& Medicinal Chemistry Letters 23, 1212-1216.

Pike K. G., Morris J., Ruston L., et al. 2015. Discovery of AZD3147: A Potent, Selective Dual Inhibitor of mTORC1 and mTORC2. Journal of Medicinal Chemistry 58, 2326-2349.

Prioretti L., Avilan L., Carriere F., Montane M. H., Field B., Gregori G., Menand B., Gontero B. 2017. inhibition of TOR in the model diatom Phaeodactylum tricornutum promotes a get-fat growth regime. Algal Research 26, 265-274.

Ren M., Venglat P., Qiu S., et al. 2012. Target of rapamycin signaling regulates metabolism, growth, and life span in Arabidopsis. The Plant Cell 24, 4850-4874.

Rexin D., Meyer C., Robaglia C., Veit B. 2015. TOR signalling in plants. Biochemical Journal 470, 1-14.

Ricaud L., Proux C., Renou J. P., Pichon O., Fochesato S., Ortet P., Montane M. H. 2007. ATM-mediated transcriptional and developmental responses to gamma-rays in Arabidopsis. PLoS One 2, e430.

Rousseau A., Bertolotti A. 2016. An evolutionarily conserved pathway controls proteasome homeostasis. Nature 536, 184-+.

Roustan V., Weckwerth W. 2018. Quantitative Phosphoproteomic and System-Level Analysis of TOR Inhibition Unravel Distinct Organellar Acclimation in Chlamydomonas reinhardtii. Frontiers in Plant Science 9.

Sabatini D. M. 2017. Twenty-five years of mTOR: Uncovering the link from nutrients to growth. Proceedings of the National Academy of Sciences, USA 114, 11818-11825.

Salem M. A., Li Y., Bajdzienko K., Fisahn J., Watanabe M., Hoefgen R., Schottler M. A., Giavalisco P. 2018. RAPTOR Controls Developmental Growth Transitions by Altering the Hormonal and Metabolic Balance. Plant Physiology 177, 565-593.

Sarbassov D. D., Ali S. M., Sengupta S., Sheen J. H., Hsu P. P., Bagley A. F., Markhard A. L., Sabatini D. M. 2006. Prolonged rapamycin treatment inhibits mTORC2 assembly and Akt/PKB. Molecular Cell 22, 159-168.

Saxton R. A., Sabatini D. M. 2017. mTOR Signaling in Growth, Metabolism, and Disease. Cell 168, 960-976.

Schepetilnikov M., Dimitrova M., Mancera-Martinez E., Geldreich A., Keller M., Ryabova L. A. 2013. TOR and S6K1 promote translation reinitiation of uORF-containing $m$ RNAs via phosphorylation of elF3h. EMBO Journal 32, 1087-1102.

Schepetilnikov M., Kobayashi K., Geldreich A., Caranta C., Robaglia C., Keller M., Ryabova L. A. 2011. Viral factor TAV recruits TOR/S6K1 signalling to activate reinitiation after long ORF translation. EMBO Journal 30, 1343-1356.

Schepetilnikov M., Makarian J., Srour O., Geldreich A., Yang Z., Chicher J., Hammann P., Ryabova L. A. 2017. GTPase ROP2 binds and promotes activation of target of rapamycin, TOR, in response to auxin. EMBO Journal 36, 886-903.

Shaik A., Bhakuni R., Kirubakaran S. 2018. Design, Synthesis, and Docking Studies of New Torin2 Analogs as Potential ATR/mTOR Kinase Inhibitors. Molecules 23.

Shi L., Wu Y., Sheen J. 2018. TOR signaling in plants: conservation and innovation. Development 145.

Sigal N. H., Dumont F. J. 1992. Cyclosporin A, FK-506, and rapamycin: pharmacologic probes of lymphocyte signal transduction. Annual Review of Immunology 10, 519-560.

Silvera D., Ernlund A., Arju R., Connolly E., Volta V., Wang J., Schneider R. J. 2017. mTORC1 and -2 Coordinate Transcriptional and Translational Reprogramming in Resistance to DNA Damage and Replicative Stress in Breast Cancer Cells. Molecular and Cellular Biology 37.

Simioni C., Cani A., Martelli A. M., Zauli G., Tabellini G., McCubrey J., Capitani S., Neri L. M. 2014. Activity of the novel mTOR inhibitor Torin-2 in B-precursor acute lymphoblastic leukemia and its therapeutic potential to prevent Akt reactivation. Oncotarget 5, 10034-10047.

Slotkin E. K., Patwardhan P. P., Vasudeva S. D., de Stanchina E., Tap W. D., Schwartz G. K. 2015. MLN0128, an ATP-competitive mTOR kinase inhibitor with potent in vitro and in vivo antitumor activity, as potential therapy for bone and soft-tissue sarcoma. Molecular Cancer Therapeutics 14, 395-406. 
Smithson L. J., Gutmann D. H. 2016. Proteomic analysis reveals GIT1 as a novel mTOR complex component critical for mediating astrocyte survival. Genes and Development 30, 1383-1388.

Song Y., Zhao G., Zhang X., et al. 2017. The crosstalk between Target of Rapamycin (TOR) and Jasmonic Acid (JA) signaling existing in Arabidopsis and cotton. Scientific Reports 7, 45830.

Sormani R., Yao L., Menand B., Ennar N., Lecampion C., Meyer C., Robaglia C. 2007. Saccharomyces cerevisiae FKBP12 binds Arabidopsis thaliana TOR and its expression in plants leads to rapamycin susceptibility. BMC Plant Biology 7, 26.

Soto-Burgos J., Bassham D. C. 2017. SnRK1 activates autophagy via the TOR signaling pathway in Arabidopsis thaliana. Plos One 12.

Sparks C. A., Guertin D. A. 2010. Targeting mTOR: prospects for mTOR complex 2 inhibitors in cancer therapy. Oncogene 29, 3733-3744.

Stan R., Mclaughlin M. M., Cafferkey R., Johnson R. K., Rosenberg M., Livi G. P. 1994. Interaction between Fkbp12-Rapamycin and Tor Involves a Conserved Serine Residue. Journal of Biological Chemistry 269, 3202732030.

Sturgill T. W., Hall M. N. 2009. Activating mutations in TOR are in similar structures as oncogenic mutations in PI3KCalpha. ACS Chemical Biology 4, 999-1015.

Suttangkakul A., Li F., Chung T., Vierstra R. D. 2011. The ATG1/ATG13 protein kinase complex is both a regulator and a target of autophagic recycling in Arabidopsis. The Plant Cell 23, 3761-3779.

Tavares M. R., Pavan I. C., Amaral C. L., Meneguello L., Luchessi A. D., Simabuco F. M. 2015. The S6K protein family in health and disease. Life Sciences 131, 1-10.

Thoreen C. C. 2017. The molecular basis of mTORC1-regulated translation. Biochemical Society Transactions 45, 213-221.

Thoreen C. C., Kang S. A., Chang J. W., et al. 2009. An ATP-competitive mammalian target of rapamycin inhibitor reveals rapamycin-resistant functions of mTORC1. Journal of Biological Chemistry 284, 8023-8032.

Thoreen C. C., Sabatini D. M. 2009. Rapamycin inhibits mTORC1, but not completely. Autophagy 5, 725-726. van Dam T. J. P., Zwartkruis F. J. T., Bos J. L., Snel B. 2011. Evolution of the TOR Pathway. Journal of Molecular Evolution 73, 209-220.

Velazquez A. F. C., Jackson W. T. 2018. So Many Roads: the Multifaceted Regulation of Autophagy Induction. Molecular and Cellular Biology 38.

Vespa L., Vachon G., Berger F., Perazza D., Faure J. D., Herzog M. 2004. The immunophilin-interacting protein AtFIP37 from Arabidopsis is essential for plant development and is involved in trichome endoreduplication. Plant Physiology 134, 1283-1292.

Vezina C., Kudelski A., Sehgal S. N. 1975. Rapamycin (Ay-22,989), a New Antifungal Antibiotic .1. Taxonomy of Producing Streptomycete and Isolation of Active Principle. Journal of Antibiotics 28, 721-726.

Walters H. E., Cox L. S. 2018. mTORC Inhibitors as Broad-Spectrum Therapeutics for Age-Related Diseases. International Journal of Molecular Science 19.

Wang P., Mugume Y., Bassham D. C. 2018a. New advances in autophagy in plants: Regulation, selectivity and function. Seminars in Cell \& Developmental Biology 80, 113-122.

Wang P., Zhao Y., Li Z., et al. 2018b. Reciprocal Regulation of the TOR Kinase and ABA Receptor Balances Plant Growth and Stress Response. Molecular Cell 69, 100-112 e106.

Wang R., Sunchu B., Perez V. I. 2017. Rapamycin and the inhibition of the secretory phenotype. Experimental Gerontology 94, 89-92.

Werth E. G., McConnell E. W., Couso Lianez I., Perrine Z., Crespo J. L., Umen J. G., Hicks L. M. 2018. Investigating the effect of target of rapamycin kinase inhibition on the Chlamydomonas reinhardtii phosphoproteome: from known homologs to new targets. New Phytologist.

Wicker L. S., Boltz R. C., Jr., Matt V., Nichols E. A., Peterson L. B., Sigal N. H. 1990. Suppression of B cell activation by cyclosporin A, FK506 and rapamycin. European Journal of Immunology 20, 2277-2283.

Xiong F., Dong P., Liu M., et al. 2016. Tomato FK506 Binding Protein 12KD (FKBP12) Mediates the Interaction between Rapamycin and Target of Rapamycin (TOR). Frontiers in Plant Science 7, 1746. 
Xiong F., Zhang R., Meng Z., et al. 2017a. Brassinosteriod Insensitive 2 (BIN2) acts as a downstream effector of the Target of Rapamycin (TOR) signaling pathway to regulate photoautotrophic growth in Arabidopsis. New Phytologist 213, 233-249.

Xiong M. N., Zhu Z. P., Tian S. W., et al. 2017b. Conditional ablation of Raptor in the male germline causes infertility due to meiotic arrest and impaired inactivation of sex chromosomes. FASEB Journal 31, 3934-3949.

Xiong Y., McCormack M., Li L., Hall Q., Xiang C., Sheen J. 2013. Glucose-TOR signalling reprograms the transcriptome and activates meristems. Nature 496, 181-186.

Xiong Y., Sheen J. 2012. Rapamycin and glucose-target of rapamycin (TOR) protein signaling in plants. Journal of Biological Chemistry 287, 2836-2842.

Xu Q., Liang S. P., Kudla J., Luan S. 1998. Molecular characterization of a plant FKBP12 that does not mediate action of FK506 and rapamycin. The Plant Journal 15, 511-519.

Yoo Y. J., Kim H., Park S. R., Yoon Y. J. 2017. An overview of rapamycin: from discovery to future perspectives. Journal of Industrial Microbiology \& Biotechnology 44, 537-553.

Yu K., Shi C., Toral-Barza L., et al. 2010. Beyond rapalog therapy: preclinical pharmacology and antitumor activity of WYE-125132, an ATP-competitive and specific inhibitor of mTORC1 and mTORC2. Cancer Research 70, 621-631.

Yu K., Toral-Barza L., Shi C., et al. 2009. Biochemical, cellular, and in vivo activity of novel ATP-competitive and selective inhibitors of the mammalian target of rapamycin. Cancer Research 69, 6232-6240.

Zhang R., Meng Z., Zhou T., Deng Y., Feng L., Wang Y., Sun G., Guo S., Ren M. 2013. ScFKBP12 bridges rapamycin and AtTOR in Arabidopsis. Plant Signaling \& Behavior 8, e26115.

Zhao J., Zhai B., Gygi S. P., Goldberg A. L. 2015. mTOR inhibition activates overall protein degradation by the ubiquitin proteasome system as well as by autophagy. Proceedings of the National Academy of Sciences, USA 112, $15790-15797$.

Zhao J. H., Garcia G. A., Goldberg A. L. 2016. Control of proteasomal proteolysis by mTOR. Nature 529, E1-E2. Zheng Y., Jiang Y. 2015. mTOR Inhibitors at a Glance. Molecular and cellular pharmacology 7, 15-20. 


\section{Tables}

1021

1022

1023

1024

1025

1026

1027

Table 1. Concentrations of rapamycin and different asTORis inhibiting TOR kinase activity (IC50), mammalian cells proliferation (IC50), WT plant root and leaf growth, and WT green

alga and diatom proliferation. Values were obtained with different in vitro kinase assays as well as different proliferation assays described in the references. Cell lines are embryonic or cancerous. Range of values was from different mammalian cell lines in the same article. Concentration unit is nM. Note that rapamycin never fully inhibits non-cancerous mammalian cell proliferation, growth of roots and leaves of $A$. thaliana seedlings or of the unicellular green alga $C$. reinhardtii.

\begin{tabular}{|c|c|c|c|c|c|c|}
\hline $\begin{array}{l}\text { Inhibitor / } \\
\text { original } \\
\text { paper on } \\
\text { mammalian } \\
\text { cells }\end{array}$ & $\begin{array}{l}\text { IC50 or } \\
\text { EC50 in } \\
\text { vitro } \\
\text { TOR } \\
\text { kinase }\end{array}$ & $\begin{array}{l}\text { IC50 } \\
\text { mammalian } \\
\text { cell } \\
\text { proliferation } \\
\left(\text { MEFs }^{\mathrm{a}} /\right. \\
\text { cancerous } \\
\text { cells }^{\mathrm{b}} \text { ) }\end{array}$ & $\begin{array}{l}\text { IC50 } \\
A . \quad \text { thaliana } \\
\text { root } \\
\text { lengthening } \\
\text { c/d }\end{array}$ & $\begin{array}{l}\text { Estimated } \\
\text { doses for } A \text {. } \\
\text { thaliana leaf } \\
\text { size reduction }\end{array}$ & $\begin{array}{l}\text { Estimated } \\
\text { IC50 } \\
\text { C. reinhardtii }\end{array}$ & $\begin{array}{l}\text { IC50 } \\
\text { P. tricornutum }\end{array}$ \\
\hline Rapamycin & & $\begin{array}{l}10-500 \\
<1-20,000\end{array}$ & $\begin{array}{l}\text { No effect up } \\
\text { to } 10,000^{c / d} \\
\text { impaired } \\
\text { solubility } \\
\text { beyond that }\end{array}$ & nd & $\begin{array}{l}\text { Couple of } \\
\text { doses } 100-500\end{array}$ & $\begin{array}{l}\text { Single dose, } \\
\text { slight effect at } \\
10,000\end{array}$ \\
\hline $\begin{array}{l}\text { PP242 } \\
\text { (Feldman et } \\
\text { al., 2009) }\end{array}$ & 8 & 1000 & nd & nd & nd & nd \\
\hline $\begin{array}{l}\text { Torin1 } \\
\text { (Thoreen et } \\
\text { al., 2009) }\end{array}$ & $1-10$ & $10-250$ & $\begin{array}{l}>1,000 \\
\text { impaired } \\
\text { solubility } \\
\text { beyond that }\end{array}$ & nd & $\begin{array}{l}\text { No dose } \\
\text { response, used } \\
\text { at } 500\end{array}$ & nd \\
\hline $\begin{array}{l}\text { KU-0063794 } \\
\text { (Garcia- } \\
\text { Martinez et } \\
\text { al., 2009) }\end{array}$ & 10 & 1,200 & $5-6,000$ & nd & nd & nd \\
\hline $\begin{array}{l}\text { WYE-354 } \\
(\mathrm{Yu} \text { et al., } \\
2009)\end{array}$ & 5 & $200-2,000$ & 2,000 & nd & nd & nd \\
\hline $\begin{array}{l}\text { Torin2 } \\
\text { (Liu et al., } \\
\text { 2013) }\end{array}$ & $0.25-10$ & $13-200$ & 500 & nd & nd & nd \\
\hline
\end{tabular}




\begin{tabular}{|l|l|l|l|l|l|l|}
\hline $\begin{array}{l}\text { AZD-8055 } \\
\text { (Chresta et } \\
\text { al., 2012) }\end{array}$ & 2.5 & 50 & 500 & $\begin{array}{l}20 \mu 1 \text { of 7,500 } \\
\text { to 30,000 nM } \\
\text { per 1 cm-wide } \\
\text { leaf and shoot } \\
\text { apex }\end{array}$ & $\begin{array}{l}\text { No dose } \\
\text { response, } \\
\text { routinely used } \\
\text { at 500 }\end{array}$ & $4000-6000$ \\
\hline $\begin{array}{l}\text { WYE- } \\
\begin{array}{l}\text { (25132 } \\
\text { (WYE-132) } \\
(\text { Yu et al., } \\
\text { 2010) }\end{array}\end{array}$ & 0.19 & $24-145$ & 200 & nd & nd & $<5,000$ \\
\hline
\end{tabular}

1028

1029

1030

1031

1032

1033

1034

1035

1036

1037

1038

1039

1040

1041

1042

1043

1044

1045

1046

1047

a (Thoreen et al., 2009); ${ }^{\mathrm{b}}$ (Guertin and Sabatini, 2007; Huang et al., 2003; Mukhopadhyay et al., 2016); ${ }^{\mathrm{c}}$ (Montane and Menand, 2013); ${ }^{\mathrm{d}}$ (Ren et al., 2012), note that growth IC50 of lines overexpressing yeast FKBP12 is observed with ca. 500-1,000 nM rapamycin, ${ }^{\mathrm{e}}$ Leaves of 3 weeks old plants grown on soil and under long days (16h) were rubbed with drops of AZD-8055 and grown for 6 days before scoring growth inhibition (Ouibrahim et al., 2015). ${ }^{\mathrm{f}}$ (Crespo et al., 2005; Juppner et al., 2018; Roustan and Weckwerth, 2018); ${ }^{\mathrm{g}}$ (Prioretti et al., 2017).

\section{Figures legends}

Figure 1: Efficacy of rapamycin and the asTORi AZD-8055 on growth and proliferation of mammalian cells, plants and algae. Dose response curves of rapamycin (A, C, E) and of AZD-8055 $(\mathbf{B}, \mathbf{D}, \mathbf{F})$ on mouse cancerous cells $(\mathbf{A}-\mathbf{B})$, Arabidopsis root growth $(\mathbf{C}-\mathbf{D})$ and $C$. reinhardtii growth (E-F). Note that mouse embryonic fibroblasts (non-cancerous) are also partially inhibited by rapamycin but completely by Torin1 (Thoreen et al., 2009). Plant dose response to rapamycin is shown for Wild Type (WT) and plant overexpressing A. thaliana (At)-, Human (Hs)-, or yeast (BP12)FKBP12 (C). For A. thaliana, dose response with AZD-8055 is shown for the WT and the TOR/tor-1 
1055

1056

1057

1058

1059

1060

1061

1062

1063

1064 1065 heterozygote, which has higher sensitivity to this asTORi due to haploinsufficiency of TOR. In all species, the efficacy of growth inhibition by rapamycin is never maximal (ca. 50\%) while it is maximal by AZD-8055. From (Giubellino et al., 2013) (A, B), (Deng et al., 2016) (C), (Montane and Menand, 2013) (D), (Juppner et al., 2018) (E) and (Imamura et al., 2016) (F). Figures and images are reproduced with permission of Oxford Academic (A, B), Wiley Online Library (E) and Taylor \& Francis $(\mathbf{F})$ or were originally published under the Creative Commons Attribution License $(\mathbf{C}, \mathbf{D})$.

\section{Figure 2: Formula of main asTORis discussed in this review.}

For original publication of each inhibitor, see text and Table 1. Drawing was done with the $\mathrm{ACD} /$ ChemSketch freeware.

Figure 3: The quality of mTORC1 substrates determines their sensitivity to rapamyin, asTORis and nutrients availability. Poor mTORC1 substrates like S6K1 T389, 4EBP1 S65 and also GRB10 S476 are inhibited by rapamycin and partial amino acid depletion. In the other hand, strong mTORC1 targets, including ULK1 S758, 4E-BP1-T37/46, but also GRB10 S150 and PRAS40 S183, are resistant to rapamycin but not to asTORis or complete starvation. From data of (Kang et al., 2013). 
fully active

$100 \%$ aminoacids
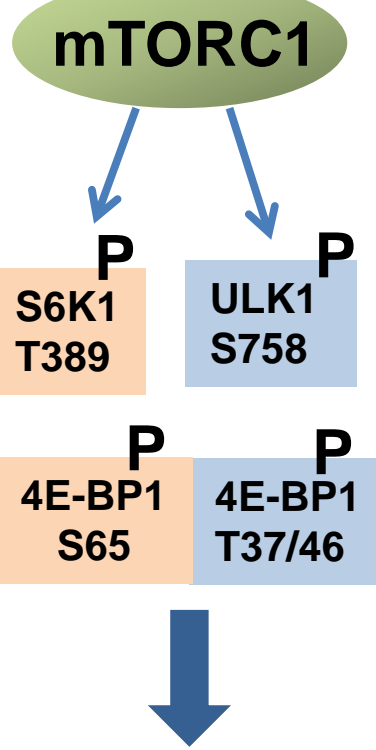

growth partial inhibition

20\% aminoacids or rapamycin

\section{mTORC1}

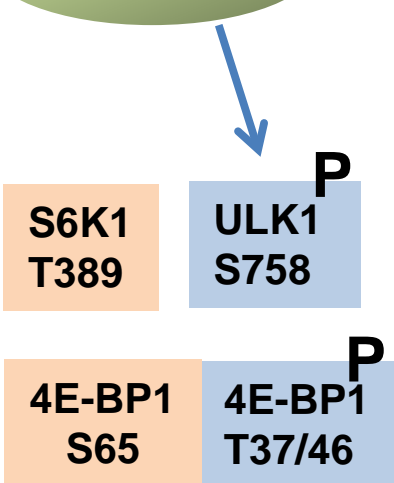

partial growth strong inhibition

$0 \%$ aminoacids or asTORis

\section{mTORC1}

S6K1

ULK1

T389

S758

4E-BP1 4E-BP1 S65 T37/46

no growth strong mTORC1 targets

poor mTORC1 targets 